\title{
Robert Winkler(1) \\ Geometrically exact shell theory from a hierarchical perspective
}

Received: 20 September 2018 / Revised: 20 December 2018 / Published online: 19 February 2019

(C) The Author(s) 2019

\begin{abstract}
A hierarchic approach for the derivation of an infinite series of nonlinear $\ell$ th-order shell theories from three-dimensional continuum mechanics based on a polynomial series expansion of the displacement field is recapitulated. Imposing the static constraints that second- and higher-order moments vanish, a 'firstorder' shell theory is obtained without employing any kinematic constraints or geometric approximations. In particular, it is shown in full generality that, within the same theoretical framework, this static assumption, on the one hand, and a common Reissner-Mindlin-type kinematic assumption, on the other hand, lead to the same theory, for which the attribute geometrically exact is adopted from the literature. This coincidence can be interpreted as a theoretical justification for the heuristic Reissner-Mindlin assumption. Further, the unexpected but unavoidable appearance of transverse moment components (residual drill moments) is addressed and analysed. Feasible assumptions are formulated which allow to separate these drill components from the remaining balance equations without affecting the equilibrium of the standard static variables. This leads to a favourable structure of the component representation of balance equations in the sense that they formally coincide with the ones of linear shear-deformable shell theory. Finally, it is shown that this result affects the interpretation of applied boundary moments.
\end{abstract}

\section{Introduction}

During the past decades, essentially two types of shear-deformable shell theories and related finite element implementations have become widely accepted. These have been attributed as geometrically exact [31] and as statically exact [4], respectively. A related terminology is adopted here. The first well-known attempt to offer a comprehensive theoretical treatment of the shell topic has been undertaken by Naghdi [21]. He differentiates between direct and derived approaches to obtain a shell theory. In the former, a shell is considered a priori as a two-dimensional continuum whose kinematics is described by a Cosserat surface, i.e., a surface equipped at each point with one or more director vectors. For a recent interpretation of the direct approach and further reading, see [9]. In the latter, the governing equations are derived from the ones of three-dimensional continuum mechanics via a dimensional reduction based on a series expansion (with respect to the transverse coordinate) of the displacement field. In [19] a mixed approach is proposed: Firstly, the balance equations governing the static variables of the shell theory are derived from three-dimensional force and moment balance. Next, a strain energy functional is assumed, and the kinematic variables are defined to be the energetically conjugate counterparts of the static ones. Finally, the constitutive equations are merely postulated in a way to obtain a specifically appropriate theory. Without applying any kinematic constraint, this approach leads to a 'statically exact' theory in the sense that the shell balance equations are exact implications of their three-dimensional

R. Winkler $(\bowtie)$

Institute of Mechatronics, University of Innsbruck, Technikerstrasse 13, 6020 Innsbruck, Austria

Tel.: +43-512-507-62752

E-mail: Robert.Winkler@uibk.ac.at 
counterparts. So-called drill moments (or: drilling couples) evolve from this formulation in a natural way [4]. Inevitably, information is lost in the course of the dimensional reduction such that the deformed geometry of the shell body is not uniquely determined by the kinematic variables. In this sense, the theory is not 'geometrically exact'. In contrast, on the basis of a Reissner-Mindlin-type kinematic constraint (that is, roughly speaking, truncating the expansion of the displacement field after the linear term), a 'geometrically exact' theory is obtained if no other geometric approximations are introduced. Within the scope of this approximation, the geometry of the deformed shell body is exactly determined by the kinematic variables. It is not 'statically exact' in the sense that the shell stresses are merely accurate to a certain level of approximation. ${ }^{1}$ Although the mixed approach is commonly linked with the statically exact theory, also the original approach to the geometrically exact theory [31] fits into this scheme. To summarize, in the context of the geometrically exact theory, kinematic assumptions are employed for the derivation of both, the static as well as the kinematic relations from the three-dimensional theory. Applying the same assumptions to the strain energy expression of the three-dimensional continuum, the strain energy of the shell is reproduced exactly. The loss of information in the course of the dimensional reduction can be attributed clearly to the application of kinematic constraints. In contrast, the static and kinematic relations of the statically exact theory do not involve any approximations. The corresponding loss of information is attributed to the approximative character of the constitutive law.

The first modern treatment of shell theory, i.e., the first one which consequently applies tensor calculus, has been given in the well-known monograph of Green and Zerna [12]. It can be considered as a geometrically linear version of the geometrically exact theory. Their followers, Basar and Krätzig [1], have presented a comprehensive treatment of the linear theory. The exposition of the geometrically nonlinear formulation contained in [1] is restricted to reduced kinematics obeying the Kirchhoff-Love constraint, see also [2]. In this line, the first fully elaborate nonlinear theory is contained in [31] which is the first part of a prominent series of articles on geometrically exact shell element formulations authored by Simo and co-workers. The exposition of shell theory in the monograph [38] is closely related to Simo's original formulation. Naghdi has introduced a systematic approach for the derivation of shell theories from three-dimensional continuum mechanics [21]. Based on a series expansion of the displacement field, an infinite hierarchy of $\ell$ th-order balance and kinematic equations inter-relating $\ell$ th with $(\ell \pm 1)$ th-order stress resultants and strain variables, respectively, are rigorously derived from three-dimensional continuum mechanics. As long as the series expansion is valid, no information is lost in the course of the dimensional reduction. Difficulties arise when a related procedure is applied to the chosen three-dimensional constitutive relation. It turns out that, even in the simplest case, the $\ell$ th-order stress resultants depend on strain variables and their gradients of all orders. Strictly speaking, the (nolinear) hierarchical approach therefore leads to a nonlocal characteristic of the constitutive model. An elaboration of the linear theory is contained in [29]. An earlier attempt to obtain hierarchic shell theories, based on an expansion utilizing Legendre polynomials, is exposed in the monograph [35]. Apart from this, it appears that the general theory has not been pursued further in the literature. Truncating the series expansion at a certain order $\ell$ poses the question which terms to adopt for the constitutive relation in order to obtain a consistent $\ell$ th-order approximation. In particular, Naghdi [21] considers a first-order approximation. This is done, however, not in a consequent way. A first-order accurate shell strain energy expression is postulated rather than derived from the three-dimensional expression. Consistent first-order approximations are discussed in [25,34], e.g., In the context of plate theory, a comprehensive definition of approximation levels has been introduced by Kienzler [17]. ${ }^{2}$ Related to this, a consistent second-order plate theory, which—in this terminology—refers to a shear-deformable theory, is discussed and contrasted with a number of other 'higher-order' theories in $[18,28]$. The complexity of the problem grows exponentially when increasing the order of approximation. Correspondingly, most higher-order approaches are devoted to special applications such as laminated, sandwich, or piezoelectric shells. Details and further references can be found, for example, in $[11,23,36]$, respectively. A mathematically rigorous treatment of the process of dimensional reduction is explicated in the well-known monographs of Ciarlet [6,7].

Nowadays, most elaborate shell finite element formulations rely on either of the here discussed theories. The geometrically exact theory is the basis of formulations involving five nodal degrees of freedom (dofs), i.e., two (out-of-plane) rotation parameters [31,37] whereas the statically exact approach naturally involves six nodal dofs, i.e., three rotational parameters (including a drilling, i.e., in-plane, rotation) [4,5]. An alternative branch of shell theories and related element formulations, which intrinsically rely on three rotational parameters, originates from [13] and has been developed further in [15,16,39]. Considerable efforts have been made to

\footnotetext{
1 The exact meanings of 'geometrically exact', 'statically/dynamically exact', and 'kinematically exact' are not unique throughout the literature. Here these terms are used in a way which fits best to the perception of the present work.

${ }^{2}$ Note that the definition of the order of a consistent approximation proposed in [17] differs from the one introduced in Sect. 4.
} 
combine the conceptual simplicity of the geometrically exact theory with the practical and numerical advantages of a six dofs finite element formulation via the application of a constraint condition which forces the drilling degree of freedom to coincide with the in-plane material rotation [10,41]. Formulations involving a rotation constraint are verified with high reliability [40]. This is not valid to the same extent for formulations relying on the statically exact theory.

In spite of the vast variety of literature on the topic, some aspects still deserve additional attention: The subsets of literature on either type of theory more or less coexist without substantial reference to each other. From a theoretical point of view, it is tempting to investigate the inter-relation of both approaches in more detail. From a practical point of view, it turns out that a better understanding of both theories can be exploited to improve the performance of statically exact shell elements. Regarding the geometrically exact theory for its own, the following research questions are identified:

(i) How can the geometrically exact shell theory be embedded in the systematic approach of Naghdi?

(ii) Is there a theoretical justification for the heuristic Reissner-Mindlin assumption, in particular, for geometric nonlinearity?

(iii) The nonlinear balance equations are usually stated in a vector format. Thereby the unavoidable existence of transverse (= drill) moment components is masked. Strictly speaking, the equilibrium can not be fulfilled exactly at the boundary if the external moments are applied tangent to the reference surface. Has this observation any relevance?

The present paper provides detailed answers to questions (i)-(iii). The exposition comes along with some preparatory work which is intended to be used in a subsequent treatise on the statically exact theory and its inter-relation to other shell theories. The article is organized as follows: A brief description in terms of differential geometry of what is considered a shell body is given in Sect. 2. Different shell coordinate systems are introduced, which play a central role for the present work. The relevant foundations of nonlinear shell kinematics are also stated there. In Sect. 3, a hierarchical shell theory is elaborated on the basis of a polynomial series expansion of the displacement field. In Sect. 4, a first-order approximation is derived by neglecting second- and higher-order moments. This is followed by a detailed analysis of the balance equations in Sect. 5 . In particular, the roles of different coordinate systems, of additional approximative assumptions, such as planestress and thinness assumptions, and of so-called residual drill moments are discussed in some detail. As a central result, it is shown in Sect. 6 that the first-order approximation formally coincides with the original geometrically exact shell theory. In Sect. 7, the basic results are summarized and relevant conclusions are drawn. For a better understanding of the mathematical notation, the relevant fundamentals of tensor algebra and analysis are collected in the "Appendix."

\section{Shell geometry and basic kinematics}

To enhance the readability of the text, notations and principles of tensor calculus and differential geometry are summarized in the "Appendix". In Sect. 2.1, the geometry of a shell body is analysed. The exposition aims at a most beneficial approach for the treatment of exact shell kinematics and thus deviates in some aspects from standard expositions of the topic. Instead of placing specific references throughout the text, it is generally referred to [30] for a concise treatment of vector and tensor calculus and to [8] concerning the beginnings of differential geometry. As usual, the following conventions (most of them related to the so-called Ricci calculus [26]) are adopted:

- Surface indices are denoted by Greek letters $\alpha, \beta, \mu, \nu, \ldots=1,2$

- Spatial indices are denoted by Latin letters $i, j, k, \ldots=1,2,3$

- Superscript indices are contravariant, subscript indices are covariant

- Dummy indices are used for function arguments, $\mathbf{a}_{3}\left(\xi^{\alpha}\right) \equiv \mathbf{a}_{3}\left(\xi^{1}, \xi^{2}\right)$, etc.

- Vectors and tensors are denoted by boldface letters

- Einstein's summation convention holds for pairings of co- and contravariant indices.

\subsection{Geometry of the shell body}

Given a reference surface $\mathcal{A}$, which is spanned by the position vectors $\overline{\mathbf{x}}\left(\xi^{\alpha}\right)$, see A.2, a shell body $\mathcal{B}$ is considered to be spanned by the position vectors

$$
\mathbf{x}\left(\xi^{\alpha}, \xi\right)=\overline{\mathbf{x}}\left(\xi^{\alpha}\right)+\zeta\left(\xi^{\alpha}, \xi\right), \quad \xi^{-} \leq \xi \leq \xi^{+},
$$


with $\zeta\left(\xi^{\alpha}, 0\right)=\mathbf{0}$ and $\zeta\left(\xi^{\alpha}, \xi\right)$, for $\xi \neq 0$, not being tangent to the reference surface. For given $\left(\xi^{1}, \xi^{2}\right)$, the vector function $\mathbf{x}(\xi)=\overline{\mathbf{x}}+\zeta(\xi)$ can be imagined as a parameterization of the transverse material fibre passing through the point $\overline{\mathbf{x}}$ of the reference surface. In principle, any of these fibres can constitute an arbitrary smooth curve as long as the function is sufficiently differentiable with respect to $\xi^{\alpha}$ and $\xi$. There are no additional $a$ priori requirements concerning the nature of the transverse coordinate $\xi$.

Since the surface normal vector $\mathbf{a}^{3}=\mathbf{a}_{3}$ is a unit vector by definition, the coordinate

$$
\zeta^{3}=\mathbf{a}^{3} \cdot \zeta
$$

measures the distance from the reference surface along $\mathbf{a}_{3}$. It is of course possible to apply a parameter transformation such that $\xi=\zeta^{3}$. Then, $t=\xi^{+}-\xi^{-}$becomes the shell thickness. For the subsequent work, $\xi$ and $\zeta^{3}$ are not to be identified necessarily. Occasionally, for a convenient application of index notation, it will be more convenient to write synonymously $\xi^{3}$ instead of just $\xi$, see (31), for example.

A natural choice for the in-plane basis vectors is (Appendix A.2)

$$
\mathbf{g}_{\alpha}=\mathbf{x}_{, \alpha}=\mathbf{a}_{\alpha}+\zeta_{, \alpha} .
$$

Deviating from a common practice, the transverse basis vector is here defined with respect to $\zeta^{3}$ instead of $\xi$,

$$
\mathbf{g}_{3}=\mathbf{x}_{, 3}=\zeta_{, 3} \equiv \frac{\partial \zeta}{\partial \zeta^{3}} .
$$

Differentiating (2) with respect to $\zeta^{3}$ yields

$$
\mathbf{a}^{3} \cdot \mathbf{g}_{3}=1
$$

Introducing a transverse shear variable $\gamma_{\beta}\left(\zeta^{3}\right)=\mathbf{a}_{\beta} \cdot \mathbf{g}_{3}\left(\zeta^{3}\right)$, one can write

$$
\mathbf{g}_{3}=\mathbf{a}_{3}+\gamma^{\beta} \mathbf{a}_{\beta} \text {. }
$$

This choice of the transverse basis vector leads to several beneficial features of the following exposition. It is emphasized that (6) does not rely on any approximative assumption. It is a consequence of the special choice of the transverse parameter $\zeta^{3}$. At the reference surface,

$$
\overline{\mathbf{g}}_{\alpha}:=\left.\mathbf{g}_{\alpha}\right|_{\xi=0}=\mathbf{a}_{\alpha}
$$

but, in general,

$$
\overline{\mathbf{g}}_{3}:=\left.\mathbf{g}_{3}\right|_{\xi=0} \neq \mathbf{a}_{3} .
$$

That is, two different covariant basis systems appear at the reference surface: The triad $\left(\mathbf{a}_{i}\right)$ and $\left(\overline{\mathbf{g}}_{i}\right)$ will be called the surface orthonormal frame and the skew director frame, respectively (Fig. 1). Their contravariant counterparts are the systems $\left(\mathbf{a}^{i}\right)$, defined in A.2, and

$$
\overline{\mathbf{g}}^{i}=\frac{1}{2} \bar{\epsilon}^{i j k} \overline{\mathbf{g}}_{j} \times \overline{\mathbf{g}}_{k}
$$

Due to $(5)$,

$$
\sqrt{\bar{g}}=\left(\overline{\mathbf{g}}_{1} \times \overline{\mathbf{g}}_{2}\right) \cdot \overline{\mathbf{g}}_{3}=\left(\mathbf{a}_{1} \times \mathbf{a}_{2}\right) \cdot \mathbf{a}_{3}=\sqrt{a}
$$

and thus $\bar{\epsilon}^{\alpha \beta 3}=\sqrt{\bar{g}} \epsilon(\alpha, \beta, 3)=\epsilon^{\alpha \beta}$. The $\epsilon$ tensors and the permutation symbol $\epsilon(\cdot)$ are defined in A.2. Consequently, in analogy with (198),

$$
\overline{\mathbf{g}}_{3} \times \overline{\mathbf{g}}_{\alpha}=\epsilon_{\alpha \beta} \overline{\mathbf{g}}^{\beta}, \quad \overline{\mathbf{g}}_{\alpha} \times \overline{\mathbf{g}}_{\beta}=\epsilon_{\alpha \beta} \overline{\mathbf{g}}^{3},
$$

and, in particular,

$$
\overline{\mathbf{g}}^{3}=\mathbf{a}^{3}
$$


It follows from (6) that

$$
\overline{\mathbf{g}}^{\beta}=\mathbf{a}^{\beta}-\bar{\gamma}^{\beta} \mathbf{a}_{3}
$$

where $\bar{\gamma}^{\beta}=\left.\gamma^{\beta}\right|_{\xi=0}$. Any spatial vector $\mathbf{v}$ can now be decomposed with respect to the surface orthonormal frame, $\mathbf{v}=v^{\beta} \mathbf{a}_{\beta}+v^{3} \mathbf{a}_{3}=v_{\beta} \mathbf{a}^{\beta}+v_{3} \mathbf{a}^{3}$ or, alternatively, with respect to the skew director frame, $\mathbf{v}=$ $v^{\bar{\beta}} \overline{\mathbf{g}}_{\beta}+v^{3} \overline{\mathbf{g}}_{3}=v_{\bar{\beta}} \overline{\mathbf{g}}^{\beta}+v_{\overline{3}} \overline{\mathbf{g}}^{3}$. The corresponding components are related to each other via

$$
\left.\begin{array}{ll}
v^{\bar{\beta}}=v^{\beta}-\bar{\gamma} \beta v^{3}, & v_{\bar{\beta}}=v_{\beta} \\
v^{\overline{3}}=v^{3}=v_{3}, & v_{\overline{3}}=v_{3}+\bar{\gamma}^{\beta} v_{\beta}
\end{array}\right\} .
$$

The spatial Christoffel symbols $\gamma_{j i}^{k}=\left.\mathbf{g}^{k} \cdot \mathbf{g}_{j, i}\right|_{\xi=0}$ at the reference surface obtain a manageable structure,

$$
\begin{aligned}
& \bar{\gamma}_{\beta \alpha}^{\mu}=\overline{\mathbf{g}}^{\mu} \cdot \overline{\mathbf{g}}_{\beta, \alpha}=\Gamma_{\beta \alpha}^{\mu}-\bar{\gamma}^{\mu} b_{\beta \alpha}, \\
& \bar{\gamma}_{3 \alpha}^{\beta}=\overline{\mathbf{g}}^{\beta} \cdot \overline{\mathbf{g}}_{3, \alpha}=-b_{\alpha}^{\beta}+\left.\bar{\gamma}^{\beta}\right|_{\alpha}-\bar{\gamma}^{\beta} \bar{\gamma}^{\mu} b_{\mu \alpha}, \\
& \bar{\gamma}_{\beta \alpha}^{3}=\overline{\mathbf{g}}^{3} \cdot \overline{\mathbf{g}}_{\beta, \alpha}=b_{\beta \alpha}, \\
& \bar{\gamma}_{3 \alpha}^{3}=\overline{\mathbf{g}}^{3} \cdot \overline{\mathbf{g}}_{3, \alpha}=\bar{\gamma}^{\mu} b_{\mu \alpha} .
\end{aligned}
$$

The surface Christoffel symbols $\Gamma_{\beta \alpha}^{\mu}$ are defined in A.2. It is noted that the $\bar{\gamma}_{j i}^{k}$ in (15.1-4) are tensor components, which is not the case for Christoffel symbols in general. This is again a consequence of the chosen parameterization and is the reason why coordinate systems $\left(\mathbf{a}_{i}\right)$ and $\left(\overline{\mathbf{g}}_{i}\right)$ are equally applicable for the formulation of a shell theory. With respect to the surface orthonormal frame, the covariant derivatives are

$$
\mathbf{a}^{\beta} \cdot \mathbf{v}_{, \alpha}=\left.v^{\beta}\right|_{\alpha}-b_{\alpha}^{\beta} v^{3}, \quad \mathbf{a}_{\beta} \cdot \mathbf{v}_{, \alpha}=\left.v_{\beta}\right|_{\alpha}-b_{\beta \alpha} v_{3},
$$

in analogy with (209) and

$$
\mathbf{a}^{3} \cdot \mathbf{v}_{, \alpha}=v_{, \alpha}^{3}+b_{\alpha \beta} v^{\beta}=v_{3, \alpha}+b_{\alpha}^{\beta} v_{\beta}=\mathbf{a}_{3} \cdot \mathbf{v}_{, \alpha} .
$$

In terms of the skew director frame,

$$
\left.\begin{array}{ll}
\overline{\mathbf{g}}^{\beta} \cdot \mathbf{v}_{, \alpha}=v^{\bar{\beta}} \|_{\alpha}+\bar{\gamma}_{3 \alpha}^{\beta} v^{3}, & \overline{\mathbf{g}}_{\beta} \cdot \mathbf{v}_{, \alpha}=v_{\bar{\beta}} \|_{\alpha}=\mathbf{a}^{\beta} \cdot \mathbf{v}_{, \alpha} \\
\overline{\mathbf{g}}^{3} \cdot \mathbf{v}_{, \alpha}=v^{3} \|_{, \alpha}+\bar{\gamma}_{\beta \alpha}^{3} v^{\bar{\beta}}=\mathbf{a}^{3} \cdot \mathbf{v}_{, \alpha}, & \overline{\mathbf{g}}_{3} \cdot \mathbf{v}_{, \alpha}=v_{\overline{3}} \|_{\alpha}-\bar{\gamma}_{3 \alpha}^{\mu} v_{\mu}
\end{array}\right\},
$$

where

$$
\left.\begin{array}{l}
v^{\bar{\beta}}\left\|_{\alpha}=v_{, \alpha}^{\bar{\beta}}+\bar{\gamma}_{\mu \alpha}^{\beta} v^{\bar{\mu}}, \quad v_{\bar{\beta}}\right\|_{\alpha}=v_{\beta, \alpha}-\bar{\gamma}_{\beta \alpha}^{\mu} v_{\mu}=\left.v_{\beta}\right|_{\alpha} \\
v^{3}\left\|_{, \alpha}=v_{, \alpha}^{3}+\bar{\gamma}_{3 \alpha}^{3} v^{3}, \quad v_{\overline{3}}\right\|_{\alpha}=v_{\overline{3}, \alpha}-\bar{\gamma}_{3 \alpha}^{3} v_{\overline{3}}
\end{array}\right\} .
$$

The shifter tensor $\mathbf{z}=\mathbf{g}_{k} \otimes \overline{\mathbf{g}}^{k}=z^{k}{ }_{i} \overline{\mathbf{g}}_{k} \otimes \overline{\mathbf{g}}^{i}$ transports the triad $\left(\overline{\mathbf{g}}_{i}\right)$ from the reference surface into the shell body,

$$
\mathbf{g}_{i}=z_{i}^{k} \overline{\mathbf{g}}_{k}=\mathbf{z} \cdot \overline{\mathbf{g}}_{i}
$$

Its components $z^{k}{ }_{i}\left(\zeta^{3}\right)=\overline{\mathbf{g}}^{k} \cdot \mathbf{g}_{i}$ read explicitly

$$
\left.\begin{array}{ll}
\overline{\mathbf{g}}^{\beta} \cdot \mathbf{g}_{\alpha}=\delta_{\alpha}^{\beta}+\gamma_{3 \alpha}^{\beta} \zeta^{3}+\zeta^{\beta} \|_{\alpha}=z_{\alpha}^{\beta}, & \overline{\mathbf{g}}^{\beta} \cdot \mathbf{g}_{3}=\gamma^{\beta}-\bar{\gamma}^{\beta}=z^{\beta}{ }_{3} \\
\overline{\mathbf{g}}^{3} \cdot \mathbf{g}_{\alpha}=b_{\alpha \beta} \zeta^{\beta}=z^{3}, & \overline{\mathbf{g}}^{3} \cdot \mathbf{g}_{3}=1=z^{3}{ }_{3},
\end{array}\right\}
$$

where use has been made of $\zeta^{3}, \alpha=0$ since the $\xi^{1}, \xi^{2}, \zeta^{3}$ are considered as mutually independent parameters. 


\subsection{Basic shell kinematics}

The common praxis in many texts on continuum mechanics, such as [14], to use upper case and lower case letters for variables related to the undeformed and deformed configuration, respectively, is largely adopted here. Without loss of generality, the undeformed configuration of the shell body $\mathcal{B}_{0}$ is parameterized as

$$
\mathbf{X}\left(\xi^{\alpha}, \xi\right)=\overline{\mathbf{X}}\left(\xi^{\alpha}\right)+\xi \mathbf{A}_{3}\left(\xi^{\alpha}\right), \quad \xi^{-} \leq \xi \leq \xi^{+} .
$$

The position vectors $\overline{\mathbf{X}}$ span the undeformed shell reference surface $\mathcal{A}_{0}$, and $\mathbf{A}_{3}$ denotes the corresponding unit normal vector. Since $\left\|\mathbf{A}_{3}\right\|=1$, the thickness coordinate $\xi$ is length measuring. Thus, $t=\xi^{+}-\xi^{-}$is the thickness of the undeformed shell. In the context of Sect. 2.1, $\zeta(\xi)=\xi \mathbf{A}_{3}, \zeta^{3}=\xi$, and $\zeta^{\beta}=0$. The in-plane basis vectors are defined in analogy with A.2,

$$
\mathbf{A}_{\alpha}=\overline{\mathbf{X}}_{, \alpha} .
$$

The natural basis vectors of the shell body are

$$
\mathbf{G}_{\alpha}=\mathbf{X}_{, \alpha}=\mathbf{A}_{\alpha}+\xi \mathbf{A}_{3, \alpha}, \quad \mathbf{G}_{3}=\mathbf{X}_{, 3}=\mathbf{A}_{3} .
$$

The contravariant ones are

$$
\mathbf{A}^{\alpha}=\epsilon_{0}^{\alpha \beta} \mathbf{A}_{\beta} \times \mathbf{A}_{3}, \quad \mathbf{A}^{3}=\frac{1}{2} \epsilon_{0}^{\alpha \beta} \mathbf{A}_{\alpha} \times \mathbf{A}_{\beta}=\mathbf{A}_{3}
$$

and

$$
\mathbf{G}^{i}=\frac{1}{2} \epsilon_{0}^{i j k} \mathbf{G}_{j} \times \mathbf{G}_{k}
$$

where $\epsilon_{0}^{i j k}=\frac{1}{\sqrt{G}} \epsilon(i, j, k)$ and $\epsilon_{0}^{\alpha \beta}=\frac{1}{\sqrt{A}} \epsilon(\alpha, \beta, 3)$ with $\sqrt{G}=\left(\mathbf{G}_{1} \times \mathbf{G}_{2}\right) \cdot \mathbf{G}_{3}=\left\|\mathbf{G}_{1} \times \mathbf{G}_{2}\right\|$ and $\sqrt{A}=\left\|\mathbf{A}_{1} \times \mathbf{A}_{2}\right\|$. The definition of contravariant basis vectors (26) implies

$$
\mathbf{G}^{i} \cdot \mathbf{G}_{j}=\delta_{j}^{i} .
$$

Together with the implicit function theorem, it follows that

$$
\mathbf{G}^{\alpha}=\frac{\partial \xi^{\alpha}}{\partial \mathbf{X}}, \quad \mathbf{G}^{3}=\frac{\partial \xi}{\partial \mathbf{X}} .
$$

In terms of the shifter tensor $\mathbf{Z}=Z_{\alpha}^{\beta} \mathbf{A}_{\beta} \otimes \mathbf{A}^{\alpha}$,

$$
\mathbf{G}_{\alpha}=Z_{\alpha}^{\beta} \mathbf{A}_{\beta}=\mathbf{Z} \cdot \mathbf{A}_{\alpha} .
$$

The components $Z_{\alpha}^{\beta}$ are symmetric,

$$
Z_{\alpha}^{\beta}=\delta_{\alpha}^{\beta}-\xi B_{\alpha}^{\beta}
$$

with $B_{\alpha}^{\beta}=-\mathbf{A}_{3} \cdot \mathbf{A}_{\beta}$ being the components of the curvature tensor.

The parameterization of the deformed shell body $\mathcal{B}_{\tau}$, Eq. (1), is chosen such that

$$
\mathbf{x}\left(\xi^{\alpha}, \xi\right)=\boldsymbol{\phi}_{\tau}\left(\mathbf{X}\left(\xi^{\alpha}, \xi\right)\right) \text {. }
$$

The vector function $\boldsymbol{\phi}_{\tau}: \mathcal{B}_{0} \rightarrow \mathcal{B}_{\tau}$ maps the undeformed body $\mathcal{B}_{0}$ onto its deformed configuration $\mathcal{B}_{\tau}$ at the time $\tau$. All definitions and expressions of Sect. 2.1 are adopted here for the geometric description of the deformed shell configuration. The deformation gradient is written as

$$
\mathbf{F}(\mathbf{X}, \tau)=\frac{\partial \boldsymbol{\phi}_{\tau}}{\partial \mathbf{X}}=\frac{\partial \mathbf{x}}{\partial \xi^{k}} \otimes \frac{\partial \xi^{k}}{\partial \mathbf{X}}=\mathbf{g}_{k}^{c} \otimes \mathbf{G}^{k} .
$$

Correspondingly, the basis vectors

$$
\mathbf{F} \cdot \mathbf{G}_{\alpha}=\mathbf{g}_{\alpha}^{c}=\frac{\partial \mathbf{x}}{\partial \xi^{\alpha}}, \quad \mathbf{F} \cdot \mathbf{G}_{3}=\mathbf{g}_{3}^{c}=\frac{\partial \mathbf{x}}{\partial \xi} .
$$

are convected ones (Fig. 1). It will turn out to be advantageous to switch from the convected frame $\left(\mathbf{g}_{i}^{c}\right)$ to the triad 


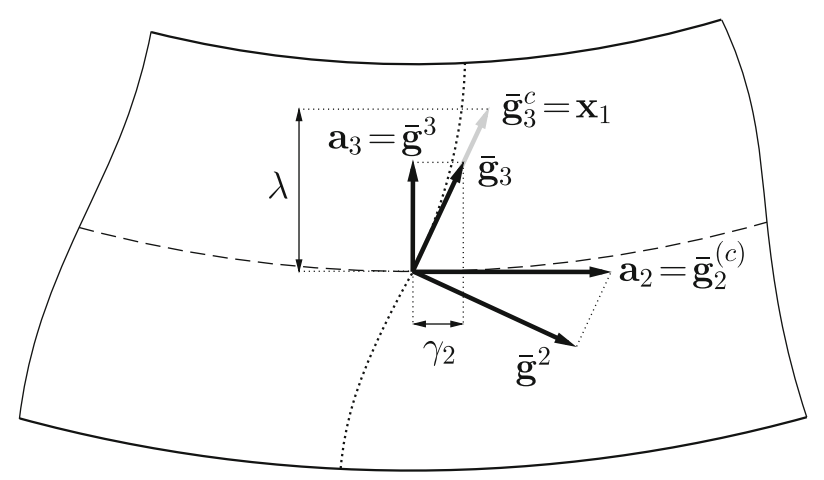

Fig. 1 Two-dimensional schematic illustration of the covariant basis vectors $\mathbf{a}_{2}, \mathbf{a}_{3}$ (surface orthogonal frame), $\overline{\mathbf{g}}_{2}, \overline{\mathbf{g}}_{3}$ (skew director frame), and $\overline{\mathbf{g}}_{2}^{c}, \overline{\mathbf{g}}_{3}^{c}$ (convected frame). Amongst their contravariant counterparts, only $\overline{\mathbf{g}}^{2}, \overline{\mathbf{g}}^{3}$ are depicted for the sake of clarity. Note the coincidences which occur in general: $\mathbf{a}_{\alpha}=\overline{\mathbf{g}}_{\alpha}=\overline{\mathbf{g}}_{\alpha}^{c}$ and $\mathbf{a}_{3}=\mathbf{a}^{3}=\overline{\mathbf{g}}^{3}$

$$
\mathbf{g}_{\alpha}=\mathbf{g}_{\alpha}^{c}, \quad \mathbf{g}_{3}=\frac{\partial \mathbf{x}}{\partial \zeta^{3}},
$$

with the defining property (5). Introducing the thickness stretch

$$
\lambda=\mathbf{a}^{3} \cdot \mathbf{g}_{3}^{c}=\mathbf{a}^{3} \cdot \frac{\partial \zeta}{\partial \xi}=\frac{\partial \zeta^{3}}{\partial \xi},
$$

one has

$$
\mathbf{g}_{3}^{c}=\frac{\partial \zeta}{\partial \xi}=\frac{\partial \zeta}{\partial \zeta^{3}} \frac{\partial \zeta^{3}}{\partial \xi}=\lambda \mathbf{g}_{3}
$$

see Fig. 1. The parameter $\xi$ will be called a material coordinate (it is length-measuring with respect to the material, i.e., undeformed, configuration) and $\zeta^{3}$ a spatial coordinate (it is length-measuring with respect to the spatial, i.e., deformed, configuration).

\section{Elements of a hierarchical shell theory}

To proceed, a hierarchic approach for the derivation of shell balance equations and kinematic relations is presented. It is a basic characteristic of this procedure that no approximative assumptions are applied a priori. It is intended to reproduce the essence of Naghdy's laborious "derivation from the three-dimensional theory" [21] with some modifications and to give it an easily readable and condensed shape. The formulation is based on the notion that any smooth displacement field of the shell body can be expanded with respect to the material thickness coordinate $\xi$,

$$
\mathbf{U}\left(\xi^{\alpha}, \xi\right)=\sum_{\ell=0}^{\infty} \xi^{\ell} \mathbf{u}_{\ell}\left(\xi^{\alpha}\right)
$$

As long as it is not intended to truncate this series at a certain term, the expansion (36) does not impose any kinematic restriction to the theory. Accordingly, the coordinates of a material point in the deformed configuration can be written as

$$
\mathbf{x}=\mathbf{X}+\mathbf{U}=\mathbf{X}_{0}+\mathbf{u}_{0}+\xi\left(\mathbf{A}_{3}+\mathbf{u}_{1}\right)+\xi^{2} \mathbf{u}_{2}+\cdots=\sum_{\ell=0}^{\infty} \xi^{\ell} \mathbf{x}_{\ell},
$$

with $\mathbf{x}_{0}=\mathbf{X}_{0}+\mathbf{u}_{0}, \mathbf{x}_{1}=\mathbf{A}_{3}+\mathbf{u}_{1}$, and $\mathbf{x}_{\ell}=\mathbf{u}_{\ell}$ for $\ell=2,3, \ldots$ Comparing expressions (1) and (37),

$$
\overline{\mathbf{x}}=\mathbf{x}_{0}, \quad \zeta=\sum_{\ell=1}^{\infty} \xi^{\ell} \mathbf{x}_{\ell}
$$




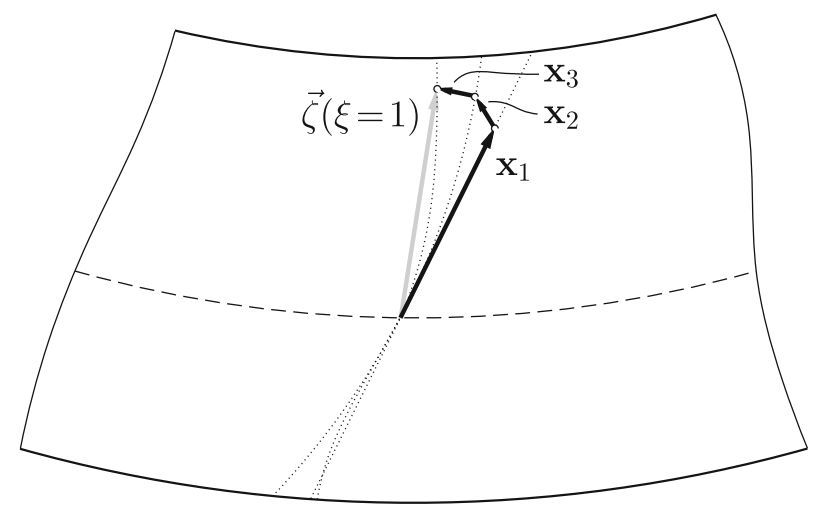

Fig. 2 Schematic visualization of expansion (38.2). The deformed transverse material fibre is illustrated as a superposition of a linear $\left(\mathbf{x}_{1}\right)$, a quadratic $\left(\mathbf{x}_{2}\right)$, and a cubic $\left(\mathbf{x}_{3}\right)$ deflection

see Fig. 2.

For the subsequent derivations, it is beneficial to make use of the convected basis vectors $\mathbf{g}_{k}^{c}=\mathbf{x}_{, k}$,

$$
\left.\begin{array}{l}
\mathbf{g}_{\alpha}=\mathbf{a}_{\alpha}+\xi \mathbf{x}_{1, \alpha}+\xi^{2} \mathbf{x}_{2, \alpha}+\cdots=\mathbf{a}_{\alpha}+\sum_{\ell=1}^{\infty} \xi^{\ell} \mathbf{x}_{\ell, \alpha} \\
\mathbf{g}_{3}=\mathbf{x}_{1}+2 \xi \mathbf{x}_{2}+3 \xi^{2} \mathbf{x}_{3}+\cdots=\sum_{\ell=1}^{\infty} \xi^{\ell-1} \ell \mathbf{x}_{\ell}
\end{array}\right\} .
$$

In the present Section, the superscript ' $c$ ' will be dropped for notational convenience as long as there is no risk of confusion. The virtual counterparts $\delta \mathbf{g}_{k}=\delta \mathbf{u}_{, k}$ are

$$
\delta \mathbf{g}_{\alpha}=\delta \mathbf{u}_{0, \alpha}+\sum_{\ell=1}^{\infty} \xi^{\ell} \delta \mathbf{u}_{\ell, \alpha}, \quad \delta \mathbf{g}_{3}=\sum_{\ell=1}^{\infty} \ell \xi^{\ell-1} \delta \mathbf{u}_{\ell} .
$$

\subsection{Hierarchical balance of forces}

To derive the shell balance equations and boundary conditions, the principle of virtual displacements (PVD) is taken as a basis [14],

$$
\delta W_{\text {int }}=\delta W_{\text {ext }},
$$

with the virtual work of external body forces $\mathbf{f}$ and surface tractions being

$$
\delta W_{\mathrm{ext}}=\int_{\mathcal{B}} \mathbf{f} \cdot \delta \mathbf{u} \mathrm{d} V+\int_{\mathcal{A}^{+}} \mathbf{t}^{+} \cdot \delta \mathbf{u} \mathrm{d} A^{+}+\int_{\mathcal{A}^{-}} \mathbf{t}^{-} \cdot \delta \mathbf{u} \mathrm{d} A^{+}+\int_{\mathcal{S}} \mathbf{t}^{s} \cdot \delta \mathbf{u} \mathrm{d} S .
$$

Therein, the boundary of the shell body has been split into top, bottom, and lateral surfaces, $\mathcal{A}^{+}, \mathcal{A}^{-}$, and $\mathcal{S}$ with applied surface tractions $\mathbf{t}^{+}, \mathbf{t}^{-}$, and $\mathbf{t}^{s}$, respectively. The volume element and the area element at the reference surface in the deformed configuration are

$$
\mathrm{d} V=\sqrt{g} \mathrm{~d} \xi^{1} \mathrm{~d} \xi^{2} \mathrm{~d} \xi=z(\xi) \mathrm{d} \xi \mathrm{d} A, \quad \mathrm{~d} A=\sqrt{a} \mathrm{~d} \xi^{1} \mathrm{~d} \xi^{2},
$$

respectively. The area elements at top/bottom and lateral surfaces are

$$
\mathrm{d} A^{ \pm}=z\left(\xi^{ \pm}\right) \mathrm{d} A, \quad \mathrm{~d} S=z^{s}(\xi) \mathrm{d} \xi \mathrm{d} s,
$$


respectively, with $\mathrm{d} s=\sqrt{a_{\alpha \beta} \mathrm{d} \xi^{\alpha} \mathrm{d} \xi^{\beta}}$ being the arc length element at the reference surface. Gram's determinant $\sqrt{g}$ is defined in (189), $z=\operatorname{det} \mathbf{z}=\sqrt{g / a}$ is the determinant of shifter tensor $\mathbf{z}$, Eq. (20), and

$$
z^{s}(\xi)=\sqrt{\frac{g_{\alpha \beta} \mathrm{d} \xi^{\alpha} \mathrm{d} \xi^{\beta}}{a_{\alpha \beta} \mathrm{d} \xi^{\alpha} \mathrm{d} \xi^{\beta}}} .
$$

The virtual work of internal forces, i.e., the virtual strain energy, is favourably written in terms of the first Piola-Kirchhoff stress tensor and deformation gradient $\mathbf{F}$,

$$
\delta W_{\text {int }}=\int_{\mathcal{B}_{0}} \mathbf{P}: \delta \mathbf{F} \mathrm{d} V_{0}
$$

where $\mathrm{d} V_{0}=\sqrt{G} \mathrm{~d} \xi^{1} \mathrm{~d} \xi^{2} \mathrm{~d} \xi$ is the volume element in the undeformed configuration. Both, Cauchy's stress tensor $\sigma$ as well as the first Piola-Kirchhoff stress tensor $\mathbf{P}$ are relevant for the further derivation,

$$
\boldsymbol{\sigma}=\sigma^{i k} \mathbf{g}_{i} \otimes \mathbf{g}_{k}, \quad \mathbf{P}=J \sigma^{i k} \mathbf{g}_{i} \otimes \mathbf{G}_{k}
$$

with $J=\operatorname{det} \mathbf{F}$. Introducing the spatial traction vectors

$$
\mathbf{s}^{k}=\sigma \cdot \mathbf{g}^{k}=\sigma^{i k} \mathbf{g}_{i}=\frac{1}{J} \mathbf{P} \cdot \mathbf{G}^{k}
$$

the stress tensors can be written as

$$
\boldsymbol{\sigma}=\mathbf{s}^{k} \otimes \mathbf{g}_{k}, \quad \mathbf{P}=J \mathbf{s}^{k} \otimes \mathbf{G}_{k} .
$$

Expressing the virtual counterpart of the deformation gradient $\mathbf{F}=\mathbf{g}_{k} \otimes \mathbf{G}^{k}$ as

$$
\delta \mathbf{F}=\delta \mathbf{u}_{, k} \otimes \mathbf{G}^{k},
$$

the strain energy (46) becomes

$$
\delta W_{\text {int }}=\int_{\mathcal{B}_{0}} \mathbf{s}^{k} \cdot \delta \mathbf{u}_{, k} J \mathrm{~d} V_{0}=\int_{\mathcal{B}} \mathbf{s}^{k} \cdot \delta \mathbf{u}_{, k} \mathrm{~d} V .
$$

Nesting surface and thickness integrals such that the volume integral in (51) becomes

$$
\delta W_{\text {int }}=\int_{\mathcal{A}}\left[\frac{1}{\sqrt{a}} \int_{\zeta^{-}}^{\zeta^{+}} \sqrt{g} \mathbf{s}^{k} \cdot \delta \mathbf{u}_{, k} \mathrm{~d} \xi\right] \mathrm{d} A
$$

and expanding

$$
\mathbf{s}^{k} \cdot \delta \mathbf{u}_{, k}=\mathbf{s}^{\alpha} \cdot \delta \mathbf{u}_{0, \alpha}+\sum_{\ell=1}^{\infty}\left(\xi^{\ell} \mathbf{s}^{\alpha} \cdot \delta \mathbf{u}_{\ell, \alpha}+\ell \xi^{\ell-1} \mathbf{s}^{3} \cdot \delta \mathbf{u}_{\ell}\right)
$$

the $\ell$ th-order moment vectors

$$
\mathbf{m}_{\ell}^{\alpha}=\frac{1}{\sqrt{a}} \int_{\zeta^{-}}^{\zeta^{+}} \sqrt{g} \xi^{\ell} \mathbf{s}^{\alpha} \mathrm{d} \xi, \quad \mathbf{m}_{\ell}^{3}=\frac{1}{\sqrt{a}} \int_{\zeta^{-}}^{\zeta^{+}} \sqrt{g} \xi^{\ell} \mathbf{s}^{3} \mathrm{~d} \xi
$$

emerges in

$$
\delta W_{\mathrm{int}}=\int_{\mathcal{A}}\left[\mathbf{m}_{0}^{\alpha} \cdot \delta \mathbf{u}_{0, \alpha}+\sum_{\ell=1}^{\infty}\left(\mathbf{m}_{\ell}^{\alpha} \cdot \delta \mathbf{u}_{\ell, \alpha}+\ell \mathbf{m}_{\ell-1}^{3} \cdot \delta \mathbf{u}_{\ell}\right)\right] \mathrm{d} A .
$$

The rightmost integral in (42),

$$
\int_{\mathcal{S}} \mathbf{t}^{s} \cdot \delta \mathbf{u} \mathrm{d} S=\int_{\partial \mathcal{A}} \sum_{\ell=1}^{\infty} \mathbf{M}_{\ell} \cdot \delta \mathbf{u}_{\ell}
$$


gives rise to the definition of applied boundary moments

$$
\mathbf{M}_{\ell}=\int_{\zeta^{-}}^{\zeta^{+}} z^{s}(\xi) \xi^{\ell} \mathbf{t}^{s} \mathrm{~d} \xi
$$

Transforming the volume integral of (42) in line with (52) and introducing the surface moments of external forces

$$
\mathbf{p}_{\ell}=\frac{1}{\sqrt{a}} \int_{\zeta^{-}}^{\zeta^{+}} \sqrt{g} \xi^{\ell} \mathbf{f} \mathrm{d} \xi+\frac{1}{\sqrt{a}}\left[\sqrt{g^{+}}\left(\xi^{+}\right)^{\ell} \mathbf{t}^{+}+\sqrt{g^{-}}\left(\xi^{-}\right)^{\ell} \mathbf{t}^{-}\right]
$$

one obtains

$$
\delta W_{\mathrm{ext}}=\int_{\mathcal{A}} \sum_{\ell=0}^{\infty} \mathbf{p}_{\ell} \cdot \delta \mathbf{u}_{\ell} \mathrm{d} A+\int_{\partial \mathcal{A}} \sum_{\ell=0}^{\infty} \mathbf{M}_{\ell} \cdot \delta \mathbf{u}_{\ell} \mathrm{d} s .
$$

Making use of the product rule,

$$
\mathbf{m}_{\ell}^{\alpha} \cdot \delta \mathbf{u}_{\ell, \alpha}=\frac{1}{\sqrt{a}}\left(\sqrt{a} \mathbf{m}_{\ell}^{\alpha} \cdot \delta \mathbf{u}_{\ell}\right)_{, \alpha}-\frac{1}{\sqrt{a}}\left(\sqrt{a} \mathbf{m}_{\ell}^{\alpha}\right)_{, \alpha},
$$

the surface divergence theorem (61),

$$
\int_{\mathcal{A}} \frac{1}{\sqrt{a}}\left(\sqrt{a} y^{\alpha}\right)_{, \alpha} \mathrm{d} A=\int_{\partial \mathcal{A}} y^{\alpha} v_{\alpha} \mathrm{d} s
$$

can be applied for $y^{\alpha}=\mathbf{m}_{\ell}^{\alpha} \cdot \delta \mathbf{u}_{\ell}$ to reformulate (55). The integrand on the left-hand side is the divergence of surface vector $\mathbf{y}=y^{\alpha} \mathbf{a}_{\alpha}$, and $v_{\alpha}=\mathbf{a}^{\alpha} \cdot \boldsymbol{v}$ are the components of the outward normal $\boldsymbol{v}$ of the boundary curve $\partial \mathcal{A}$. Putting all together, the PVD can be given the shape

$$
\begin{aligned}
& -\int_{\mathcal{A}} \sum_{\ell=0}^{\infty}\left[\frac{1}{\sqrt{a}}\left(\sqrt{a} \mathbf{m}_{\ell}^{\alpha}\right)_{, \alpha}-\ell \mathbf{m}_{\ell-1}^{3}+\mathbf{p}_{\ell}\right] \cdot \delta \mathbf{u}_{\ell} \mathrm{d} A \\
& +\int_{\partial \mathcal{A}} \sum_{\ell=0}^{\infty}\left(v_{\alpha} \mathbf{m}_{\ell}^{\alpha}-\mathbf{M}_{\ell}\right) \cdot \delta \mathbf{u}_{\ell} \mathrm{d} s=0 .
\end{aligned}
$$

Via standard arguments of variational calculus, the PVD (62) delivers an infinite sequence of balance laws,

$$
\begin{aligned}
& \frac{1}{\sqrt{a}}\left(\sqrt{a} \mathbf{m}_{0}^{\alpha}\right)_{, \alpha}+\mathbf{p}_{0}=\mathbf{0}, \\
& \frac{1}{\sqrt{a}}\left(\sqrt{a} \mathbf{m}_{1}^{\alpha}\right)_{, \alpha}-\mathbf{m}_{0}^{3}+\mathbf{p}_{1}=\mathbf{0}, \\
& \vdots \\
& \frac{1}{\sqrt{a}}\left(\sqrt{a} \mathbf{m}_{\ell}^{\alpha}\right)_{, \alpha}-\ell \mathbf{m}_{\ell-1}^{3}+\mathbf{p}_{\ell}=\mathbf{0},
\end{aligned}
$$

as well as the static boundary conditions

$$
v_{\alpha} \mathbf{m}_{\ell}^{\alpha}=\mathbf{M}_{\ell} \text { at } \mathcal{C}_{\ell}^{m} .
$$

For a proper application of the boundary conditions, the boundary $\partial \mathcal{A}$ has to be split (for each $\ell=0,1,2, \ldots$ ) into two disjunct parts $\mathcal{C}_{\ell}^{u}$ and $\mathcal{C}_{\ell}^{m}$, such that $\partial \mathcal{A}=\mathcal{C}_{\ell}^{u} \cup \mathcal{C}_{\ell}^{m}$. At the former, the $\ell$ th-order displacements $\mathbf{u}_{\ell}^{*}$ are prescribed,

$$
\mathbf{u}_{\ell}=\mathbf{u}_{\ell}^{*} \text { at } \mathcal{C}_{\ell}^{u},
$$

(primary boundary conditions), and the $\mathbf{M}_{\ell}$ play the role of reaction moments.

It is important to note that the entity of shell balance Eq. (63) and boundary conditions (64) is equivalent to the PVD and thus to the force balance of three-dimensional continuum mechanics. Although (63.2) has the appearance of a (first-order) moment balance, they are independent of three-dimensional moment balance. 
3.2 Hierarchical balance of moments

The three-dimensional balance of moments is equivalent to the symmetry of Cauchy's stress tensor [12],

$$
\mathbf{0}=\sigma^{i j}\left(\mathbf{g}_{i} \times \mathbf{g}_{j}\right)=\mathbf{g}_{i} \times\left(\sigma^{i j} \mathbf{g}_{j}\right)=\mathbf{g}_{i} \times\left(\sigma \cdot \mathbf{g}^{i}\right) .
$$

Making use of traction vectors (48),

$$
\mathbf{g}_{\alpha} \times \mathbf{s}^{\alpha}+\mathbf{g}_{3} \times \mathbf{s}^{3}=\mathbf{0}
$$

Inserting into (39) and performing a thickness integration, $\frac{1}{\sqrt{a}} \int \sqrt{g}(\cdot) \mathrm{d} \xi$, yields

$$
\mathbf{a}_{\alpha} \times \mathbf{m}_{0}^{\alpha}=-\sum_{\ell=1}^{\infty}\left(\mathbf{x}_{\ell, \alpha} \times \mathbf{m}_{\ell}^{\alpha}+\ell \mathbf{x}_{\ell} \times \mathbf{m}_{\ell-1}^{3}\right) .
$$

Equation (68) is a necessary condition for moment balance but not a sufficient one. Via thickness integration, $\frac{1}{\sqrt{A}} \int \xi^{\ell}(\cdot) \sqrt{G} \mathrm{~d} \xi$ for $\ell=1,2, \ldots$, an infinite sequence of additional equations is obtained,

$$
\mathbf{a}_{\alpha} \times \mathbf{m}_{\ell}^{\alpha}=-\sum_{L=1}^{\infty}\left(\mathbf{x}_{L, \alpha} \times \mathbf{m}_{\ell+L}^{\alpha}+L \mathbf{x}_{L} \times \mathbf{m}_{\ell+L-1}^{3}\right) .
$$

\subsection{The kinematic variables}

The $\ell$ th-order displacement gradients $\varphi_{k}^{\ell}$, defined by

$$
\mathbf{U}_{, i}=\sum_{\ell=0}^{\infty} \xi^{\ell} \boldsymbol{\varphi}_{i}^{\ell},
$$

read explicitly

$$
\varphi_{\alpha}^{\ell}=\mathbf{u}_{\ell, \alpha}, \quad \varphi_{3}^{\ell}=(\ell+1) \mathbf{u}_{\ell+1}
$$

Thereby, the virtual strain energy (55) can be written as

$$
\delta W_{\mathrm{int}}=\int_{\mathcal{A}} \sum_{\ell=0}^{\infty} \mathbf{m}_{\ell}^{k} \cdot \delta \boldsymbol{\varphi}_{k}^{\ell} \mathrm{d} A .
$$

Concluding, $\ell$ th-order moment vectors $\mathbf{m}_{\ell}^{k}$ and displacement gradients $\varphi_{k}^{\ell}$ are work conjugate.

3.4 The component representations of balance equations

Inserting $\mathbf{s}^{k}=\sigma^{i k} \mathbf{g}_{i}$ in (54), the moment vectors read

$$
\mathbf{m}_{\ell}^{k}=\frac{1}{\sqrt{a}} \int_{\zeta^{-}}^{\zeta^{+}} \sqrt{g} \xi^{\ell} \sigma^{i k} \mathbf{g}_{i} \mathrm{~d} \xi .
$$

Their components $m_{\ell}^{i k}=\overline{\mathbf{g}}^{i} \cdot \mathbf{m}_{\ell}^{k}$ with respect to the convected frame at the reference surface, $\left(\overline{\mathbf{g}}_{i}\right)$, are

$$
m_{\ell}^{i k}=\frac{1}{\sqrt{a}} \int_{\zeta^{-}}^{\zeta^{+}} \sqrt{g} \xi^{\ell} z_{j}^{i}(\xi) \sigma^{j k} \mathrm{~d} \xi
$$


The components of the (convected) shell shifter, $z_{j}^{i}:=\overline{\mathbf{g}}^{i} \cdot \mathbf{g}_{j}$, depend on the deformed geometry and are, thus, a priori unknown functions of $\xi$,

$$
\left.\begin{array}{ll}
z^{\beta}{ }_{\alpha}=\delta_{\alpha}^{\beta}+\xi \gamma_{3 \alpha}^{\beta}+\sum_{\ell=2}^{\infty} \xi^{\ell} z_{\ell \alpha}^{\beta}, & z^{\beta}{ }_{3}=\sum_{\ell=2}^{\infty} \xi^{(\ell-1)} x_{\ell}^{\beta} \\
z^{3}{ }_{\alpha}=\xi \gamma_{3 \alpha}^{3}+\sum_{\ell=2}^{\infty} \xi^{\ell} z_{\ell \alpha}^{3}, & z^{3}{ }_{3}=1+\sum_{\ell=2}^{\infty} \xi^{(\ell-1)} x_{\ell}^{3}
\end{array}\right\}
$$

where

$$
z_{\ell \alpha}^{\beta}=x_{\ell}^{\beta}\left\|_{\alpha}+\gamma_{3 \alpha}^{\beta} x_{\ell}^{3}, \quad z_{\ell \alpha}^{3}=x_{\ell}^{3}\right\|_{\alpha}+\gamma_{\alpha \beta}^{3} x_{\ell}^{\beta} .
$$

Note that here $\mathbf{g}_{j}=\mathbf{g}_{j}^{c}$ are the convected basis vectors, $\overline{\mathbf{g}}^{i}=\left.\mathbf{g}^{i}\right|_{\xi=0}$, and $\gamma_{j, k}^{i}=\overline{\mathbf{g}}^{i} \cdot \overline{\mathbf{g}}_{j, k}$. (The bar on the $\gamma$ coefficients, which has been introduced in (15), is dropped for notational convenience.) Consequently, $\overline{\mathbf{g}}_{3}=\mathbf{x}_{1}$ and thus

$$
x_{1}^{\beta}=\overline{\mathbf{g}}^{\beta} \cdot \mathbf{x}_{1}=0, \quad x_{1}^{3}=\overline{\mathbf{g}}^{3} \cdot \mathbf{x}_{1}=1,
$$

and further, $z_{1 \alpha}^{\beta}=\gamma_{3 \alpha}^{\beta}$ and $z_{1 \alpha}^{3}=\gamma_{3 \alpha}^{3}$.

The explicit appearance of the $z^{i}{ }_{j}$ can be avoided if the expression $\mathbf{s}^{k}=\frac{1}{J} \mathbf{P} \cdot \mathbf{G}^{\alpha}$ is used instead to obtain

$$
m_{\ell}^{i \alpha}=\frac{1}{\sqrt{a}} \int_{\zeta^{-}}^{\zeta^{+}} \sqrt{G} \xi^{\ell} S_{\beta}^{\alpha}(\xi) P^{i \beta} \mathrm{d} \xi, \quad m_{\ell}^{i 3}=\frac{1}{\sqrt{a}} \int_{\zeta^{-}}^{\zeta^{+}} \sqrt{G} \xi^{\ell} P^{i 3} \mathrm{~d} \xi .
$$

Here, the components of the inverse shell shifter $\mathbf{S}=\mathbf{A}_{\alpha} \otimes \mathbf{G}^{\alpha}=S_{\alpha}^{\beta} \mathbf{A}_{\beta} \otimes \mathbf{A}^{\alpha}$,

$$
S_{\alpha}^{\beta}=\mathbf{G}^{\beta} \cdot \mathbf{A}_{\alpha}=\delta_{\alpha}^{\beta}+\xi B_{\alpha}^{\beta}+\xi^{2} B_{\mu}^{\beta} B_{\alpha}^{\mu}+\xi^{3} B_{\mu}^{\beta} B_{\nu}^{\mu} B_{\alpha}^{\nu}+\cdots,
$$

only depend on the undeformed geometry. However, the stress components $P^{i j}=\mathbf{a}^{i} \cdot \mathbf{P} \cdot \mathbf{A}^{j}$ are, in general, non-symmetric, in contrast to $J \sigma^{i j}=\mathbf{g}^{i} \cdot \mathbf{P} \cdot \mathbf{G}^{j}$, and do not feature a simple physical interpretation.

Expanding the moment vectors,

$$
\mathbf{m}_{\ell}^{\alpha}=m_{\ell}^{\beta \alpha} \overline{\mathbf{g}}_{\beta}+m_{\ell}^{3 \alpha} \overline{\mathbf{g}}_{3}, \quad \mathbf{m}_{\ell}^{3}=m_{\ell}^{\beta 3} \overline{\mathbf{g}}_{\beta}+m_{\ell}^{33} \overline{\mathbf{g}}_{3},
$$

the balance Eq. (63) gives rise to the component representations of force balance

$$
\left.\begin{array}{l}
m_{0}^{\beta \alpha} \|_{\alpha}+\gamma_{3 \alpha}^{\beta} m_{0}^{3 \alpha}+p_{0}^{\beta}=0 \\
m_{0}^{3 \alpha} \|_{\alpha}+\gamma_{3 \alpha}^{3} m_{0}^{3 \alpha}+\gamma_{\alpha \beta}^{3} m_{0}^{\beta \alpha}+p_{0}^{3}=0
\end{array}\right\}
$$

and

$$
\left.\begin{array}{l}
m_{\ell}^{\beta \alpha} \|_{\alpha}+\gamma_{3 \alpha}^{\beta} m_{\ell}^{3 \alpha}-\ell m_{\ell-1}^{\beta 3}+p_{\ell}^{\beta}=0 \\
m_{\ell}^{3 \alpha} \|_{\alpha}+\gamma_{3 \alpha}^{3} m_{\ell}^{3 \alpha}+\gamma_{\alpha \beta}^{3} m_{\ell}^{\beta \alpha}-\ell m_{\ell-1}^{33}+p_{\ell}^{3}=0
\end{array}\right\}
$$

for $\ell=1,2, \ldots$ Inserting (80) into (69) yields the component representations of moment balance,

$$
\begin{aligned}
& m_{\ell}^{3 \alpha}=\sum_{L=1}^{\infty}\left[z_{L \mu}^{3} m_{\ell+L}^{\alpha \mu}-z_{L \mu}^{\alpha} m_{\ell+L}^{3 \mu}+L\left(x_{L}^{3} m_{\ell+L-1}^{\alpha 3}-x_{L}^{\alpha} m_{\ell+L-1}^{33}\right)\right] \\
& \epsilon_{\alpha \beta} m_{\ell}^{\beta \alpha}=\sum_{L=1}^{\infty}\left(z_{L \mu}^{\beta} m_{\ell+L}^{\alpha \mu}+L x_{L}^{\beta} m_{\ell+L-1}^{\alpha 3}\right)
\end{aligned}
$$


To obtain an interpretation of these very involved formulas, they are rearranged,

$$
\left.\begin{array}{l}
m_{\ell}^{3 \alpha}-m_{\ell}^{\alpha 3}-\gamma_{3 \mu}^{3} m_{\ell+1}^{\alpha \mu} \\
=\sum_{L=2}^{\infty}\left[z_{L \mu}^{3} m_{\ell+L}^{\alpha \mu}-z_{(L-1) \mu}^{\alpha} m_{\ell+L-1}^{3 \mu}+L\left(x_{L}^{3} m_{\ell+L-1}^{\alpha 3}-x_{L}^{\alpha} m_{\ell+L-1}^{33}\right)\right] \\
\epsilon_{\alpha \beta}\left(m_{\ell}^{\beta \alpha}-\gamma_{3 \mu}^{\beta} m_{\ell+1}^{\alpha \mu}\right)=\sum_{L=2}^{\infty}\left(z_{L \mu}^{\beta} m_{\ell+L}^{\alpha \mu}+L x_{L}^{\beta} m_{\ell+L-1}^{\alpha 3}\right)
\end{array}\right\}
$$

such that the right-hand sides vanish if, for any $\ell=0,1,2, \ldots$, no moments $m_{\ell+2}^{\alpha \mu}, m_{\ell+1}^{\alpha 3}, m_{\ell+1}^{3 \alpha}$, and higherorder ones are present. In particular, for $\ell=0$, the symmetry relations of conventional shell theory are obtained (Sect. 4.3).

The geometrically linear case is obtained by setting $\overline{\mathbf{g}}_{3}=\mathbf{x}_{1}=\mathbf{A}_{3}$ and $\mathbf{x}_{2}=\mathbf{x}_{3}=\cdots=\mathbf{0}$. Then $x_{1}^{3}=1$, $z_{1 \mu}^{\beta}=-B_{\mu}^{\beta}$, and all other $x$ and $z$ components vanish. Correspondingly, Eq. (83) becomes

$$
\left.\begin{array}{rl}
m_{\ell}^{3 \alpha}-m_{\ell}^{\alpha 3} & =B_{\mu}^{\alpha} m_{\ell+1}^{3 \mu} \\
\epsilon_{\alpha \beta}\left(m_{\ell}^{\beta \alpha}+B_{\mu}^{\beta} m_{\ell+1}^{\alpha \mu}\right) & =0
\end{array}\right\} .
$$

\section{Derivation of a first-order approximation}

The approach of Sect. 3 leads to a hierarchy of $\ell$ th-order shell theories. In this context, an $\ell$ th-order theory is obtained if all $(\ell+1)$ and higher-order moments are neglected. This understanding of the approximation order is adopted here. It is important to note that several other definitions of the notion of 'order' of a shell (or plate) theory exist: In [23], amongst others, the order $\ell$ is equal to the highest-order term $\xi^{\ell} u_{\ell}^{k}$ in the thickness expansion of the displacement field. Both, Kirchhoff-Love as well as Reissner-Mindlin-type theories (this naming is adopted from [3]) are of first-order in this sense. Correspondingly, the latter are sometimes called 'first-order shear deformation (FOSD) theories'. In the context of linear plate theories, Kienzler expands the strain energy with respect to a small characteristic parameter $c^{2}$ and truncates the series after the term involving $c^{2 \ell}$ to obtain a consistent $\ell$ th-order approximation [17]. Accordingly, zeroth-, first-, and second-order approximations refer to membrane theory, Kirchhoff-Love, and Reissner-Mindlin plate theories, respectively.

The 'best linear shell theory-type A' (translated from the German) described in the monograph [1] is considered here as a prototype of a linear, geometrically exact shell theory. It is derived from the threedimensional theory under the assumption of the well-known Reissner-Mindlin constraint stating that "straight material fibres originally oriented perpendicular to the reference surface remain straight and unstretched" [20,24]. In [29], the same linear theory is obtained from a hierarchic approach. To be more exact: Postulating that second and higher-order moments vanish and that $m_{0}^{33}=0$, i.e., the 'zero transverse normal stress assumption' (Sect. 5.4), the linear counterpart of the present hierarchic approach on the one hand and the prototypical, linear theory on the other hand lead to exactly the same appearance of the PVD. In other words, exclusively static constraints, on the one hand, and exclusively kinematic constraints, on the other, lead to the same shell theory.

In the present Section, it is shown that an analogous result holds for the general nonlinear case. The prototypical nonlinear, geometrically exact shell theory originates from [31], where the corresponding PVD is derived from its three-dimensional counterpart applying the Reissner-Mindlin constraint. Here, the same expression for the PVD is obtained from the hierarchic approach applying static constraints. No kinematic assumptions or geometric approximations are required. It is emphasized that this finding does not simply result from a trivial generalization of the linear circumstances. From the present point of view, the term 'geometrically exact' gains additional justification, and its opposition to the attribute 'statically exact' (no static constraints) becomes manifest. The static assumptions turn out to be, in some sense, stronger than their kinematic counterparts, subsumed in the Reissner-Mindlin assumption: The symmetry of bending moments $m_{1}^{\beta \alpha}$ and the absence of shear force couples $m_{1}^{3 \alpha}$ (not to be confused with the drill moments $m^{3 \alpha}$ ) are a consequence of the static assumptions. In contrast, earlier formulations of the nonlinear theory consider the symmetry of the $m_{1}^{\beta \alpha}$ and the absence of the $m_{1}^{3 \alpha}$ as additional approximative assumptions. 
4.1 Balance equations

The static constraints which will lead to the desired shell theory read explicitly

$$
\mathbf{m}_{\ell}^{k}=\mathbf{0}, \quad \mathbf{p}_{\ell}=\mathbf{0} \text { and } \mathbf{M}_{\ell}^{k}=\mathbf{0}, \quad \text { for } \ell=2,3, \ldots
$$

Accordingly, only three non-trivial, vectorial balance equations out of system (63.1) remain,

$$
\frac{1}{\sqrt{a}}\left(\sqrt{a} \mathbf{m}_{0}^{\alpha}\right)_{, \alpha}+\mathbf{p}_{0}=\mathbf{0}, \quad \frac{1}{\sqrt{a}}\left(\sqrt{a} \mathbf{m}_{1}^{\alpha}\right)_{, \alpha}-\mathbf{m}_{0}^{3}+\mathbf{p}_{1}=\mathbf{0}, \quad \mathbf{m}_{1}^{3}=\mathbf{0} .
$$

In addition, the three-dimensional moment balance (69) becomes

$$
\mathbf{a}_{\alpha} \times \mathbf{m}_{0}^{\alpha}+\mathbf{x}_{1} \times \mathbf{m}_{0}^{3}+\mathbf{x}_{1, \alpha} \times \mathbf{m}_{1}^{\alpha}=\mathbf{0}
$$

for $\ell=0$, and

$$
\mathbf{a}_{\alpha} \times \mathbf{m}_{1}^{\alpha}=\mathbf{0}
$$

for $\ell=1$. Applying $\mathbf{x}_{1} \times$ from the left to (87.2) and inserting (88) yields

$$
\frac{1}{\sqrt{a}}\left(\sqrt{a} \mathbf{m}^{\alpha}\right)_{, \alpha}+\mathbf{a}_{\alpha} \times \mathbf{m}_{0}^{\alpha}+\mathbf{l}=\mathbf{0}
$$

with the resultant stress couple vector

$$
\mathbf{m}^{\alpha}=\frac{1}{\sqrt{a}} \int_{\zeta^{-}}^{\zeta^{+}} \sqrt{g} \xi^{3} \mathbf{x}_{1} \times \mathbf{s}^{\alpha} \mathrm{d} \xi^{3}=\mathbf{x}_{1} \times \mathbf{m}_{1}^{\alpha}
$$

and the resultant surface moment vector

$$
\mathbf{I}=\frac{1}{\sqrt{a}} \int_{\zeta^{-}}^{\zeta^{+}} \sqrt{g} \xi^{3} \mathbf{x}_{1} \times \mathbf{f} \mathrm{d} \xi^{3}+\frac{1}{\sqrt{a}}\left[\sqrt{g} \xi^{3} \mathbf{x}_{1} \times \mathbf{s}^{3}\right]_{t^{-}}^{t^{+}}=\mathbf{x}_{1} \times \mathbf{p}_{1}
$$

Note that in (90) the transverse normal force vector $\mathbf{m}_{0}^{3}$ has disappeared due to the balance of momentum without having imposed any additional restriction (like a 'plane stress' assumption). One is now dealing with two kinds of moment balance equations, (87.2) and (90) involving the two different sets of moment vectors $\mathbf{m}_{0}^{\alpha}$ and $\mathbf{m}^{\alpha}$, respectively.

One possibility to formulate the relevant boundary conditions out of the infinite sets of conditions (64) and (65) is given by

$$
v_{\alpha} \mathbf{m}_{0}^{\alpha}=\mathbf{M}_{0}, \quad v_{\alpha} \mathbf{m}^{\alpha}=\mathbf{L}
$$

at subsets $\mathcal{C}_{n}$ and $\mathcal{C}_{m}$, respectively, of the boundary $\partial \mathcal{A}$, and

$$
\mathbf{u}_{0}=\mathbf{u}_{0}^{*}, \quad \mathbf{x}_{1}=\mathbf{x}_{1}^{*}
$$

at $\partial \mathcal{A} \backslash \mathcal{C}_{n}$ and $\partial \mathcal{A} \backslash \mathcal{C}_{m}$, respectively. The vector of external boundary moments

$$
\mathbf{L}:=\mathbf{x}_{1} \times \mathbf{M}_{1}
$$

has been defined according to (91) and (92). 


\subsection{Force and moment components}

To analyse the component representations of the balance equations, as a first choice, the convected frame $\overline{\mathbf{g}}_{i}^{c}$ at the reference surface is employed. With $m_{0}^{\beta \alpha}=\overline{\mathbf{g}}_{c}^{\beta} \cdot \mathbf{m}_{0}^{\alpha}, m_{0}^{3 \alpha}=\overline{\mathbf{g}}_{c}^{3} \cdot \mathbf{m}_{0}^{\alpha}$, etc., the decompositions of force and moment vectors read

$$
\mathbf{m}_{0}^{\alpha}=m_{0}^{\beta \alpha} \overline{\mathbf{g}}_{\beta}^{c}+m_{0}^{3 \alpha} \overline{\mathbf{g}}_{3}^{c}, \quad \mathbf{m}_{1}^{\alpha}=m_{1}^{\beta \alpha} \overline{\mathbf{g}}_{\beta}^{c}+m_{1}^{3 \alpha} \overline{\mathbf{g}}_{3}^{c} .
$$

Since $\overline{\mathbf{g}}_{3}^{c}=\mathbf{x}_{1}$, the $m_{0}^{3 \alpha}$ components drop out from the decomposition of alternative moment vectors $\mathbf{m}^{\alpha}$,

$$
\mathbf{m}^{\alpha}=\overline{\mathbf{g}}_{3}^{c} \times \mathbf{m}_{1}^{\alpha}=m_{1}^{\beta \alpha} \overline{\mathbf{g}}_{3}^{c} \times \overline{\mathbf{g}}_{\beta}^{c}
$$

Note that

$$
m^{\beta \alpha}=\left(\overline{\mathbf{g}}_{c}^{3} \times \overline{\mathbf{g}}_{c}^{\beta}\right) \cdot \mathbf{m}^{\alpha}=\overline{\mathbf{g}}_{c}^{\beta} \cdot \mathbf{m}_{1}^{\alpha}=m_{1}^{\beta \alpha} .
$$

In contrast, the $m^{3 \alpha}=\overline{\mathbf{g}}_{c}^{3} \cdot \overline{\mathbf{m}}$ and the $m_{1}^{3 \alpha}=\overline{\mathbf{g}}_{c}^{3} \cdot \mathbf{m}_{1}^{\alpha}$ are conceptually totally different. The latter are accurately addressed as shear force couples. At a first glance, the former can be considered as 'drill moments', which is correct in the sense of moment components acting about the surface normal direction $\overline{\mathbf{g}}_{c}^{3}=\mathbf{a}^{3}$. It is not correct in the sense of 'in-plane bending moments', which is the physical meaning of 'genuine' drill moments in the context of statically exact theories. To emphasize this discrepancy, in the present context, the $m^{3 \alpha}$ are called residual drill moments (the choice of this terminology is supported by the arguments of Sect. 5.3). They will be treated further in Sect. 5.2 and subsequent ones. The external force and moment vectors are decomposed as

$$
\mathbf{p}_{0}=p_{0}^{\beta} \overline{\mathbf{g}}_{\beta}^{c}+p_{0}^{3} \overline{\mathbf{g}}_{3}^{c}, \quad \mathbf{l}=l^{\beta} \overline{\mathbf{g}}_{3}^{c} \times \overline{\mathbf{g}}_{\beta}^{c}
$$

where $l^{\beta}=\overline{\mathbf{g}}_{c}^{\beta} \cdot \mathbf{p}_{1}=p_{1}^{\beta}$ and $p_{1}^{3}$ has disappeared, as the $m^{3 \alpha}$ did.

According to (74), force and moment components read explicitly

$$
\left.\begin{array}{l}
m_{0}^{\beta \alpha}=\frac{1}{\sqrt{g}} \int_{\zeta^{-}}^{\zeta^{+}} \sqrt{g} \sigma^{\beta \alpha} \mathrm{d} \xi+\gamma_{3 \mu}^{\beta} m_{1}^{\mu \alpha}+\underbrace{x_{2}^{\beta} m_{1}^{3 \alpha}}_{=0} \\
m_{0}^{3 \alpha}=\frac{1}{\sqrt{\bar{g}}} \int_{\zeta^{-}}^{\zeta^{+}} \sqrt{g} \sigma^{3 \alpha} \mathrm{d} \xi+\gamma_{3 \mu}^{3} m_{1}^{\mu \alpha}+\underbrace{x_{2}^{\overline{3}} m_{1}^{3 \alpha}}_{=0} \\
m_{0}^{\beta 3}=\frac{1}{\sqrt{\bar{g}}} \int_{\zeta^{-}}^{\zeta^{+}} \sqrt{g} \sigma^{\beta 3} \mathrm{~d} \xi+\underbrace{\gamma_{3 \alpha}^{\beta} m_{1}^{3 \alpha}+x_{2}^{\beta} m_{1}^{33}}_{=0} \\
m_{0}^{33}=\frac{1}{\sqrt{g}} \int_{\zeta^{-}}^{\zeta^{+}} \sqrt{g} \sigma^{33} \mathrm{~d} \xi+\underbrace{\gamma_{3 \alpha}^{3} m_{1}^{3 \alpha}+x_{2}^{3} m_{1}^{33}}_{=0}
\end{array}\right\}
$$

and

$$
\left.\begin{array}{ll}
m_{1}^{\beta \alpha}=\frac{1}{\sqrt{\bar{a}}} \int_{\zeta^{-}}^{\zeta^{+}} \sqrt{g} \xi \sigma^{\beta \alpha} \mathrm{d} \xi=m^{\alpha \beta}, & m_{1}^{3 \alpha}=\frac{1}{\sqrt{\bar{g}}} \int_{\zeta^{-}}^{\zeta^{+}} \sqrt{g} \sigma^{3 \alpha} \mathrm{d} \xi=0 \\
m_{1}^{\beta 3}=\frac{1}{\sqrt{\bar{g}}} \int_{\zeta^{-}}^{\zeta^{+}} \sqrt{g} \xi \sigma^{\beta 3} \mathrm{~d} \xi=0, & m_{1}^{33}=\frac{1}{\sqrt{\bar{g}}} \int_{\zeta^{-}}^{\zeta^{+}} \sqrt{g} \xi \sigma^{33} \mathrm{~d} \xi=0
\end{array}\right\} .
$$

All terms involving second and higher powers of $\xi$ disappear due to the static assumptions (86). Due to the symmetry of the $\sigma^{\beta \alpha}$, the $m_{1}^{\beta \alpha}$ are symmetric, which is, in general, not the case for the $m_{0}^{\beta \alpha}$. The symmetric counterparts of the $m_{0}^{\beta \alpha}$ are the well-known pseudo-membrane forces of the geometrically exact theory,

$$
n^{\beta \alpha}:=m_{0}^{\beta \alpha}-\gamma_{3 \mu}^{\beta} m_{1}^{\alpha \mu}=\frac{1}{\sqrt{\bar{g}}} \int_{\zeta^{-}}^{\zeta^{+}} \sqrt{g} \sigma^{\beta \alpha} \mathrm{d} \xi=n^{\alpha \beta} .
$$


As already observed for the $m^{\beta \alpha}$, the symmetry of the $n^{\beta \alpha}$ is a direct consequence of the symmetry of the $\sigma^{j i}=\mathbf{g}^{j} \cdot \boldsymbol{\sigma} \cdot \mathbf{g}^{i}$. Similarly, the pseudo-shear force components are

$$
n^{3 \alpha}:=m_{0}^{3 \alpha}-\gamma_{3 \mu}^{3} m_{1}^{\alpha \mu}=\frac{1}{\sqrt{\bar{a}}} \int_{\zeta^{-}}^{\zeta^{+}} \sqrt{g} \sigma^{3 \alpha} \mathrm{d} \xi^{3}=m_{0}^{\alpha 3}
$$

The $n^{\beta \alpha}$ and the $n^{3 \alpha}$ can be considered as the components of the pseudo-resultant force vectors

$$
\mathbf{n}^{\alpha}:=\mathbf{m}_{0}^{\alpha}-m_{1}^{\alpha \mu} \overline{\mathbf{g}}_{3, \mu}^{c}=n^{\beta \alpha} \overline{\mathbf{g}}_{\beta}^{c}+n^{3 \alpha} \overline{\mathbf{g}}_{3}^{c} .
$$

\subsection{Component representations of balance equations}

The component representations of force balance (87.1) have already been given in (81),

$$
\left.\begin{array}{l}
m_{0}^{\beta \alpha} \|_{\alpha}+\gamma_{3 \alpha}^{\beta} m_{0}^{3 \alpha}+p_{0}^{\beta}=0 \\
m_{0}^{3 \alpha} \|_{\alpha}+\gamma_{3 \alpha}^{3} m_{0}^{3 \alpha}+\gamma_{\beta \alpha}^{3} m_{0}^{\beta \alpha}+p_{0}^{3}=0
\end{array}\right\}
$$

The ones of moment balance (90) are obtained by inserting (96.1), (97), and (99),

$$
\begin{aligned}
& m^{\beta \alpha} \|_{\alpha}+\gamma_{3 \alpha}^{3} m^{\beta \alpha}-m_{0}^{3 \beta}+l^{\beta}=0, \\
& \epsilon_{\alpha \beta}\left(m_{0}^{\beta \alpha}+\gamma_{3 \mu}^{\alpha} m^{\beta \mu}\right)=0 .
\end{aligned}
$$

The divergence is related to the metric defined by the convected frame $\left(\overline{\mathbf{g}}_{i}^{c}\right)$ at the reference surface,

$$
m_{\ell}^{\beta \alpha}\left\|_{\alpha}=\frac{1}{\sqrt{\bar{g}}}\left(\sqrt{\bar{g}} m_{\ell}^{\beta \alpha}\right)_{, \alpha}+\gamma_{\mu \alpha}^{\beta} m_{\ell}^{\mu \alpha}, \quad m_{\ell}^{3 \alpha}\right\|_{\alpha}=\frac{1}{\sqrt{\bar{g}}}\left(\sqrt{\bar{g}} m_{\ell}^{3 \alpha}\right)_{, \alpha} .
$$

Alternatively, Eq. (82) read for $\ell=1$

$$
\begin{aligned}
& m_{1}^{\beta \alpha} \|_{\alpha}+\gamma_{3 \alpha}^{\beta} m_{1}^{3 \alpha}-m_{0}^{\beta 3}+p_{1}^{\beta}=0, \\
& m_{1}^{3 \alpha} \|_{\alpha}+\gamma_{3 \alpha}^{3} m_{1}^{3 \alpha}+\gamma_{\alpha \beta}^{3} m_{1}^{\beta \alpha}-m_{0}^{33}+p_{1}^{3}=0,
\end{aligned}
$$

and for $\ell=2$

$$
m_{1}^{\beta 3}=0, \quad m_{1}^{33}=0
$$

Note that in (108.2) the unconventional quantities $m_{0}^{33}$ and $p_{1}^{3}$ are still present, which is in contrast to (106.1) where they have disappeared due to the application of (88). The three-dimensional moment balance (84) yields for $\ell=0$

$$
\begin{aligned}
& m_{0}^{3 \alpha}-m_{0}^{\alpha 3}-\gamma_{3 \mu}^{3} m_{1}^{\alpha \mu}=0 \\
& \epsilon_{\alpha \beta}\left(m_{0}^{\beta \alpha}-\gamma_{3 \mu}^{\beta} m_{1}^{\alpha \mu}\right)=0,
\end{aligned}
$$

and for $\ell=1$

$$
m_{1}^{3 \alpha}=m_{1}^{\alpha 3}, \quad \epsilon_{\alpha \beta} m_{1}^{\beta \alpha}=0 .
$$

Consequently, subtracting (106.1) from (108.1), the symmetry relation in (104) is recovered. Further, (108.2) simplifies to become

$$
\gamma_{\alpha \beta}^{3} m_{1}^{\beta \alpha}-m_{0}^{33}+p_{1}^{3}=0
$$


Equation (110.2) constitutes the symmetry relation for the pseudo-membrane forces $n^{\beta \alpha}$. Due to the antisymmetry of the $\epsilon$-tensor, Eqs. (106.2) and (110.2) are equivalent. Concluding, the moment balance earns a notably simple shape,

$$
\left.\begin{array}{rl}
m^{\beta \alpha} \|_{\alpha}-n^{3 \beta}+l^{\beta} & =0 \\
\epsilon_{\alpha \beta} n^{\beta \alpha} & =0
\end{array}\right\} .
$$

Equation (113) formally coincide with the corresponding ones of linear shell theory. It is emphasized that, apart from the static assumptions (86), no approximations have been applied so far. In contrast to (113), the force balance Eq. (106) involve the cumbersome Christoffel symbols $\gamma_{j \alpha}^{i}$ explicitly. From a practical point of view, this is not a severe drawback, since the component equations are not needed to obtain numerical solutions. It has been demonstrated in [32] that a finite element discretization can be performed advantageously on the level of the vector equations. Nevertheless, it turns out that further analysing the component equations helps to clarify some hidden aspects of the theory.

\subsection{The geometrically linear case}

Things are much less involved for the geometrically linear case: Relating the balance equations to the undeformed configuration, the basis vectors $\overline{\mathbf{g}}_{\alpha}^{c}$ and $\overline{\mathbf{g}}_{3}^{c}$ are replaced by their undeformed counterparts $\mathbf{A}_{\alpha}$ and $\mathbf{A}_{3}$, respectively, and the balance equations simplify accordingly,

$$
\begin{aligned}
& \left.m_{0}^{\beta \alpha}\right|_{\alpha}-B_{\alpha}^{\beta} m_{0}^{3 \alpha}+p_{0}^{\beta}=0, \\
& \left.m_{0}^{3 \alpha}\right|_{\alpha}+B_{\beta \alpha} m_{0}^{\beta \alpha}+p_{0}^{3}=0,
\end{aligned}
$$

and

$$
\begin{gathered}
\left.m_{1}^{\beta \alpha}\right|_{\alpha}-m_{0}^{3 \beta}+p_{1}^{\beta}=0, \\
\epsilon_{\alpha \beta}\left(m_{0}^{\beta \alpha}-B_{\mu}^{\alpha} m_{1}^{\beta \mu}\right)=0
\end{gathered}
$$

where the covariant derivative is defined with respect to the undeformed metric.

\subsection{More on symmetry relations}

The symmetry relation (106.2) refers to alternative force components

$$
\tilde{n}^{\beta \alpha}=m_{0}^{\beta \alpha}+\gamma_{3 \mu}^{\alpha} m_{1}^{\beta \mu}=\frac{1}{\sqrt{g}} \int_{\zeta^{-}}^{\zeta^{+}} \sqrt{g} \tilde{\sigma}^{\beta \alpha} \mathrm{d} \xi=\tilde{n}^{\alpha \beta}
$$

deriving from stress components $\tilde{\sigma}^{j i}:=\overline{\mathbf{a}}^{j} \cdot \sigma \cdot \overline{\mathbf{a}}^{i} \neq \sigma^{j i}$. The second equality follows from

$$
\begin{aligned}
\tilde{n}^{\beta \alpha} & =n^{\beta \alpha}+\gamma_{3 \mu}^{\beta} m_{1}^{\alpha \mu}+\gamma_{3 \mu}^{\alpha} m_{1}^{\beta \mu} \\
& =\frac{1}{\sqrt{\bar{g}}} \int_{\zeta^{-}}^{\zeta^{+}} \sqrt{g}\left(\sigma^{\beta \alpha}+\xi \gamma_{3 \mu}^{\beta} \sigma^{\alpha \mu}+\xi \gamma_{3 \mu}^{\alpha} \sigma^{\beta \mu}\right) \mathrm{d} \xi
\end{aligned}
$$

with

$$
\begin{aligned}
\tilde{\sigma}^{j i} & =z^{l}{ }_{j} z^{k}{ }_{i} \sigma^{j i}=\left[\delta_{j}^{l}+\xi \gamma_{3 j}^{l}+O\left(\xi^{2}\right)\right]\left[\delta_{i}^{k}+\xi \gamma_{3 i}^{k}+O\left(\xi^{2}\right)\right] \sigma^{j i} \\
& =\sigma^{l k}+\xi \gamma_{3 j}^{l} \sigma^{j k}+\xi \gamma_{3 i}^{k} \sigma^{i l}+O\left(\xi^{2}\right)
\end{aligned}
$$

consulting the usual arguments. 
The symmetry relation (110.1) has given rise to the definition of 'pseudo'-shear forces in (103). Alternatively, the same relation allows to define another type of 'pseudo'-shear forces,

$$
n^{\alpha 3}:=m_{0}^{\alpha 3}+\gamma_{3 \mu}^{3} m_{1}^{\alpha \mu}=\frac{1}{\sqrt{\bar{a}}} \int_{\zeta^{-}}^{\zeta^{+}} \sqrt{g} \tilde{\sigma}^{\alpha 3} \mathrm{~d} \xi^{3}=m_{0}^{3 \alpha} .
$$

Concluding, the 'pseudo'-shear forces $n^{3 \alpha}$ and $n^{\alpha 3}$ turn out to coincide mutually with their regular counterparts, i.e., $n^{3 \alpha}=m_{0}^{\alpha 3}$ and $n^{\alpha 3}=m_{0}^{3 \alpha}$.

\subsection{Membrane state of stress}

A massive simplification of balance Eqs. (105) and (106) is obtained for a membrane state of stress, i.e., for

$$
m_{1}^{\beta \alpha} \equiv 0, \quad p_{1}^{\beta} \equiv 0 .
$$

Accordingly, the moment balance (106) reduces to

$$
m_{0}^{3 \beta}=0, \quad \epsilon_{\alpha \beta} m_{0}^{\beta \alpha}=0,
$$

and the force balance (105) becomes

$$
m_{0}^{\beta \alpha} \|_{\alpha}+p_{0}^{\beta}=0, \quad b_{\beta \alpha} m_{0}^{\beta \alpha}+\bar{p}_{0}^{3}=0 .
$$

To obtain (122.2) it is noted that $\overline{\mathbf{g}}_{c}^{3}=\frac{1}{\lambda} \mathbf{a}_{3}$, see (34), and thus

$$
\gamma_{\beta \alpha}^{3}=\overline{\mathbf{g}}_{c}^{3} \cdot \mathbf{a}_{\beta, \alpha}=\frac{1}{\lambda} b_{\beta \alpha}, \quad p_{0}^{3}=\overline{\mathbf{g}}_{c}^{3} \cdot \mathbf{p}_{0}=\frac{1}{\lambda} \bar{p}_{0}^{3} .
$$

In the geometrically linear case, Eqs. (121) and (122) constitute a system of six equations governing the six unknown functions $n^{3 \alpha}$ and $n^{\beta \alpha}$ as long as at least one component of the $b_{\beta \alpha}$ is not equal to zero, i.e., for an originally curved structure. With other words, a membrane state of stress can be considered as a statically determinate problem. ${ }^{3}$ These well-known circumstances are usually considered as a corollary of conventional linear shell theory. In the hierarchical context, it becomes apparent that the membrane theory derives from three-dimensional continuum mechanics via the assumptions (120) without imposing any other restrictions like kinematic constraints or assumptions concerning second- or higher-order moments.

\section{Analysis of the first-order approximation}

\subsection{The role of transverse stretch}

The influence of transverse (i.e., thickness) stretch $\lambda$ is revealed by switching from the convected frame $\left(\overline{\mathbf{g}}_{i}^{c}\right)$ to the skew director frame $\left(\overline{\mathbf{g}}_{i}\right)$, which has been introduced in Sect. 2.1. From now on it is essential to differentiate notationally between these two triads,

$$
\begin{array}{ll}
\overline{\mathbf{g}}_{\alpha}^{c}=\overline{\mathbf{g}}_{\alpha}, & \overline{\mathbf{g}}_{3}^{c}=\lambda \overline{\mathbf{g}}_{3}=\mathbf{x}_{1}, \\
\overline{\mathbf{g}}_{c}^{\alpha}=\overline{\mathbf{g}}^{\alpha}, & \overline{\mathbf{g}}_{c}^{3}=\frac{1}{\lambda} \overline{\mathbf{g}}^{3}=\frac{1}{\lambda} \mathbf{a}^{3} .
\end{array}
$$

Further, $\sqrt{\bar{g} c}=\lambda \sqrt{\bar{g}}$. Here, $\lambda=\left.\lambda\right|_{\xi=0}$, the 'bar' is omitted for notational convenience. Vector and tensor components with respect to the convected frame will be indicated by a left-hand superscript ' $c$ ', those with respect to the skew director frame by a bar. In particular,

$$
{ }^{c} m_{0}^{\beta \alpha}=\bar{m}_{0}^{\beta \alpha}, \quad{ }^{c} m_{0}^{3 \alpha}=\frac{1}{\lambda} \bar{m}_{0}^{3 \alpha},
$$

\footnotetext{
${ }^{3}$ This is not true in general, since, in the nonlinear case, the $b_{\beta \alpha}$ are unknown functions as well.
} 


$$
{ }^{c} p_{0}^{\beta}=\bar{p}_{0}^{\beta}, \quad{ }^{c} p_{0}^{3}=\frac{1}{\lambda} \bar{p}_{0}^{3},
$$

and

$$
\begin{aligned}
& { }^{c} m_{1}^{\beta \alpha}=\bar{m}_{1}^{\beta \alpha}={ }^{c} m^{\beta \alpha}=\frac{1}{\lambda} \bar{m}^{\beta \alpha}, \quad{ }^{c} m_{1}^{3 \alpha}=\frac{1}{\lambda} \bar{m}_{1}^{3 \alpha}, \\
& { }^{c} p_{1}^{\beta}=\bar{p}_{1}^{\beta}={ }^{c}{ }^{\beta}=\frac{1}{\lambda} \bar{l}^{\beta}, \quad{ }^{c} p_{1}^{3}=\frac{1}{\lambda} \bar{p}_{1}^{3} .
\end{aligned}
$$

The Christoffel symbols with respect to the convected metric at the reference surface are

$$
\left.\begin{array}{rl}
{ }^{c} \gamma_{\beta \alpha}^{\mu} & =\overline{\mathbf{g}}_{c}^{\mu} \cdot \overline{\mathbf{g}}_{\beta, \alpha}^{c}=\gamma_{\beta \alpha}^{\mu} \\
{ }^{c} \gamma_{3 \alpha}^{\beta} & =\overline{\mathbf{g}}_{c}^{\beta} \cdot \overline{\mathbf{g}}_{3, \alpha}^{c}=\lambda \gamma_{3 \alpha}^{\beta} \\
{ }^{c} \gamma_{\beta \alpha}^{3} & =\overline{\mathbf{g}}_{c}^{3} \cdot \overline{\mathbf{g}}_{\beta, \alpha}^{c}=\frac{1}{\lambda} \gamma_{\beta \alpha}^{3} \\
{ }^{c} \gamma_{3 \alpha}^{3} & =\overline{\mathbf{g}}_{c}^{3} \cdot \overline{\mathbf{g}}_{3, \alpha}^{c}=\frac{\lambda, \alpha}{\lambda}+\gamma_{3 \alpha}^{3}
\end{array}\right\}
$$

where the $\bar{\gamma}_{j \alpha}^{k}$ are the ones given in (15). Again, the bar on the $\gamma$ coefficients will be skipped. The component equations with respect to the skew director frame,

$$
\left.\begin{array}{l}
\bar{m}_{0}^{\beta \alpha} \|_{\alpha}+\gamma_{3 \alpha}^{\beta} \bar{m}_{0}^{3 \alpha}+\bar{p}_{0}^{\beta}=0 \\
\bar{m}_{0}^{3 \alpha} \|_{\alpha}+\gamma_{3 \alpha}^{3} \bar{m}_{0}^{3 \alpha}+\gamma_{\beta \alpha}^{3 \alpha} \bar{m}_{0}^{\beta \alpha}+\bar{p}_{0}^{3}=0
\end{array}\right\}
$$

and

$$
\left.\begin{array}{l}
\bar{m}^{\beta \alpha} \|_{\alpha}+\gamma_{3 \alpha}^{3} \bar{m}^{\beta \alpha}-\bar{m}_{0}^{3 \beta}+\bar{l}^{\beta}=0 \\
\epsilon_{\alpha \beta}\left(\bar{m}_{0}^{\beta \alpha}+\gamma_{3 \mu}^{\alpha} \bar{m}^{\beta \mu}\right)=0
\end{array}\right\}
$$

formally coincide with the ones derived for the convected frame, (105) and (106), respectively. With other words, they are formally independent of thickness stretch $\lambda$. In contrast, this is not the case for the symmetry relation (110.1), which now reads

$$
\bar{m}_{0}^{3 \alpha}-\bar{x}_{1}^{3} \bar{m}_{0}^{\alpha 3}-\bar{x}_{1}^{3} \|_{\mu} \bar{m}_{1}^{\alpha \mu}=0
$$

or, with $\bar{x}_{1}^{3}=\lambda$ and $\bar{x}_{1}^{3} \|_{\mu}=\lambda, \mu+\gamma_{3 \mu}^{3} \lambda$,

$$
\bar{m}_{0}^{3 \alpha}-\lambda \bar{m}_{0}^{\alpha 3}-\left(\frac{\lambda, \mu}{\lambda}+\gamma_{3 \mu}^{3}\right) \bar{m}^{\alpha \mu}=0 .
$$

It will turn out in Sect. 6.2 that Eq. (134) fits well in the present framework in spite of its rather complicated shape.

\subsection{The role of shear deformation}

The essential structure behind the complicated shape of the balance Eqs. (131) and (132) can be carved out by another coordinate transformation, i.e., by switching from the skew director frame $\left(\overline{\mathbf{g}}_{i}\right)$ to the surface orthonormal frame $\left(\mathbf{a}_{i}\right)$, also introduced in Sect. 2.1,

$$
\begin{aligned}
& \overline{\mathbf{g}}_{\alpha}=\overline{\mathbf{g}}_{\alpha}^{c}=\mathbf{a}_{\alpha}, \quad \overline{\mathbf{g}}_{3}=\frac{1}{\lambda} \overline{\mathbf{g}}_{3}^{c}=\mathbf{a}_{3}+\gamma^{\beta} \mathbf{a}_{\beta}, \\
& \overline{\mathbf{g}}^{\alpha}=\overline{\mathbf{g}}_{c}^{\alpha}=\mathbf{a}^{\alpha}-\gamma^{\alpha} \mathbf{a}^{3}, \quad \overline{\mathbf{g}}^{3}=\lambda \overline{\mathbf{g}}_{c}^{3}=\mathbf{a}^{3},
\end{aligned}
$$


and $\sqrt{\bar{g}}=\sqrt{a}$. The 'bar' is again omitted for $\gamma^{\beta}=\left.\gamma^{\beta}\right|_{\xi=0}$. Introducing the 'non-bared' components, i.e., the components with respect to the surface orthonormal frame, $m_{0}^{k \alpha}=\mathbf{a}^{k} \cdot \mathbf{m}_{0}^{\alpha}$ and $p_{0}^{k}=\mathbf{a}^{k} \cdot \mathbf{p}_{0}^{k}$, one has

$$
\begin{aligned}
& \bar{m}_{0}^{\beta \alpha}=\overline{\mathbf{g}}^{\beta} \cdot \mathbf{m}_{0}^{\alpha}=m_{0}^{\beta \alpha}-\gamma^{\beta} m_{0}^{3 \alpha}, \quad \bar{m}_{0}^{3 \alpha}=\overline{\mathbf{g}}^{3} \cdot \mathbf{n}^{\alpha}=\mathbf{a}^{3} \cdot \mathbf{n}^{\alpha}=m_{0}^{3 \alpha}, \\
& \bar{p}_{0}^{\beta}=\overline{\mathbf{g}}^{\beta} \cdot \mathbf{p}_{0}=p_{0}^{\beta}-\gamma^{\beta} p_{0}^{3}, \quad \bar{p}_{0}^{3}=\overline{\mathbf{g}}^{3} \cdot \mathbf{p}_{0}=\mathbf{a}^{3} \cdot \mathbf{p}_{0}=p_{0}^{3} .
\end{aligned}
$$

Inserting in (131) yields

$$
\left.\begin{array}{l}
\left.m_{0}^{\beta \alpha}\right|_{\alpha}-b_{\alpha}^{\beta} m_{0}^{3 \alpha}+p_{0}^{\beta}=0 \\
\left.m_{0}^{3 \alpha}\right|_{\alpha}+b_{\beta \alpha} m_{0}^{\beta \alpha}+p_{0}^{3}=0
\end{array}\right\} .
$$

That is, the force balance equations have been given a shape which coincides formally with the one of their linear counterparts. Note that still no approximations apart from the static assumptions (86) have been made. The same result is achieved by expanding the vector Eq. (87.1) with respect to the surface orthonormal frame.

For the moment components,

$$
\begin{aligned}
& m^{\beta \alpha}=\left(\mathbf{a}^{3} \times \mathbf{a}^{\beta}\right) \cdot \mathbf{m}^{\alpha}=\left(\overline{\mathbf{g}}^{3} \times \overline{\mathbf{g}}^{\beta}\right) \cdot\left(\lambda \overline{\mathbf{g}}_{3} \times \mathbf{m}_{1}^{\alpha}\right)=\lambda \bar{m}_{1}^{\beta \alpha}=\bar{m}^{\beta \alpha}, \\
& l^{\beta}=\left(\mathbf{a}^{3} \times \mathbf{a}^{\beta}\right) \cdot \mathbf{l}=\left(\overline{\mathbf{g}}^{3} \times \overline{\mathbf{g}}^{\beta}\right) \cdot\left(\lambda \overline{\mathbf{g}}_{3} \times \mathbf{p}_{1}\right)=\lambda \bar{p}_{1}^{\beta}=\bar{l}^{\beta} .
\end{aligned}
$$

The last equality in each line follows from (128). Expanding the vector form of moment balance (90), the residual drill moments $m^{3 \alpha}$ and $l^{3}$ (see Sect. 4.2) enter the component equations,

$$
\begin{aligned}
& \left.m^{\beta \alpha}\right|_{\alpha}-m_{0}^{3 \beta}-\epsilon^{\beta \mu} b_{\alpha \mu} m^{3 \alpha}+l^{\beta}=0, \\
& \left.m^{3 \alpha}\right|_{\alpha}-\epsilon_{\beta \alpha}\left(m_{0}^{\beta \alpha}-b_{\alpha}^{\beta} m^{\gamma \alpha}\right)+l^{3}=0,
\end{aligned}
$$

The skew director frame helps to obtain a characterization of the residual drill components,

$$
\begin{aligned}
& \bar{m}^{3 \alpha}=m^{3 \alpha}=\mathbf{a}^{3} \cdot \mathbf{m}^{\alpha}=\mathbf{a}^{3} \cdot\left(\lambda \overline{\mathbf{g}}_{3} \times \mathbf{m}^{\alpha}\right)=\epsilon_{\beta \mu} \gamma^{\beta} m^{\mu \alpha}, \\
& \bar{l}^{3}=l^{3}=\mathbf{a}^{3} \cdot \mathbf{l}=\mathbf{a}^{3} \cdot\left(\lambda \overline{\mathbf{g}}_{3} \times \mathbf{p}_{1}\right)=\epsilon_{\beta \mu} \gamma^{\beta} l^{\mu} .
\end{aligned}
$$

Inserting (143) and (144) in (142.1) or, alternatively, (140) and (141) in (106), the moment balance becomes

$$
\begin{aligned}
& \left.m^{\beta \alpha}\right|_{\alpha}-\gamma^{\beta} b_{\mu \alpha} m^{\mu \alpha}+\gamma^{\mu} b_{\mu \alpha} m^{\beta \alpha}-m_{0}^{3 \beta}+l^{\beta}=0, \\
& \epsilon_{\beta \alpha}\left[m_{0}^{\beta \alpha}-b_{\mu}^{\beta} m^{\alpha \mu}-\left.\left(\gamma^{\beta} m^{\alpha \mu}\right)\right|_{\mu}-\gamma^{\beta} l^{\alpha}\right]=0 .
\end{aligned}
$$

To obtain (145.2), the Ricci lemma (218) has been considered. In contrast to the force balance (139), extra terms involving the transverse shear strains $\gamma_{\beta}$ appear in (145). Their significance will be discussed in the proximate Sections.

\subsection{The role of residual drill moments}

The component representation with respect to the surface orthogonal basis of the shell balance equations reveals the formal difference compared to their linear counterparts: This difference is represented by the residual drill moments $m^{3 \alpha}$ and $l^{3}$. Subsequently, it will be argued why they do not contribute significantly to the shell equilibrium in the context of the first-order approximation. In Sects. 5.4 and 5.5, it is demonstrated how feasible assumptions lead to the estimate

$$
\epsilon^{\beta \mu} b_{\alpha \mu} m^{3 \alpha}=\gamma^{\beta} b_{\mu \alpha} m^{\mu \alpha}-\gamma^{\mu} b_{\mu \alpha} m^{\beta \alpha} \ll m_{0}^{3 \beta}
$$




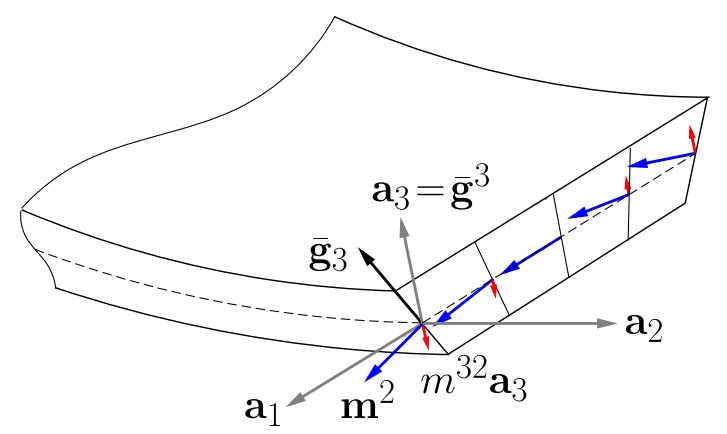

Fig. 3 Schematic visualization of the appearance of a residual drill moment $m^{32}$ ('small' vectors parallel to the normal direction $\mathbf{a}_{3}$ ) as a consequence of a bending moment $\mathbf{m}^{2}=\bar{m}^{22} \overline{\mathbf{g}}_{3} \times \mathbf{a}_{2}$ (vectors 'slightly' deviating from the in-plane direction $\mathbf{a}_{1}$ ) and a related transverse shear deformation due to the Poisson effect

such that the $m^{3 \alpha}$ drop out from (142.1),

$$
\left.m^{\beta \alpha}\right|_{\alpha}-m_{0}^{3 \beta}+l^{\beta}=0 .
$$

Considering (142.2), the argumentation is as follows: Assuming hyperelastic material behaviour, the constitutive relation for the membrane strains (Sect. 6)

$$
\varepsilon_{\beta \alpha}=\frac{1}{2}\left(\mathbf{a}_{\beta} \cdot \mathbf{a}_{\alpha}-\mathbf{A}_{\beta} \cdot \mathbf{A}_{\alpha}\right)
$$

reads

$$
\tilde{n}^{\beta \alpha}=\frac{\partial w}{\partial \varepsilon_{\beta \alpha}}
$$

with $w=w\left(\varepsilon_{\beta \alpha}\right)$ being the strain energy density. Due to the symmetry of the $\varepsilon_{\beta \alpha}$, their energetically conjugate counterparts $\tilde{n}^{\beta \alpha}$ are necessarily symmetric as well. To identify symmetric resultant force variables, only the symmetry conditions (145.2) are available. These contain derivatives and the external moment components $l^{\beta}$, deriving from internal and external drill moment components, respectively. However, a proper hyperelastic constitutive relation has to be independent of both. This discrepancy can be circumvented only by postulating that internal and external drill moment components equilibrate each other, i.e.,

$$
\left.m^{3 \alpha}\right|_{\alpha}+l^{3}=0
$$

such that (142.2), or equivalently (145.2) pass into

$$
\epsilon_{\beta \alpha}\left(m_{0}^{\beta \alpha}-b_{\mu}^{\alpha} m^{\beta \mu}\right)=0 .
$$

Concluding, the drill moment components have entirely disappeared from the moment balance Eqs. (147) and (150), which now formally coincide with their linear counterparts, as the force balance Eq. (139) does.

The corresponding physical interpretation of residual drill moments is as follows: In principle, the appearance of moment components in the transverse direction is unavoidable due to the definitions of moment vectors (91), (92), and (95) as long as a transverse shear deformation occurs; see Fig. 3. On the basis of feasible assumptions (zero normal stress, thinness, small transverse shear and bending strains, and hyperelasticity) the transverse moment components can be separated from the conventional balance equations, such that the shell equilibrium is uniquely determined by Eqs. (139), (147), (150), relating membrane forces, shear forces, and bending moments, together with the static boundary conditions

$$
v_{\alpha} m_{0}^{\beta \alpha}=M_{0}^{\beta}, \quad v_{\alpha} m_{0}^{3 \alpha}=M_{0}^{3}, \quad v_{\alpha} m^{\beta \alpha}=L^{\beta} .
$$

The transverse (= drill) moment components may simply be ignored (i.e., they need not to be in equilibrium), which is justified by the preceding argumentation. Accordingly, the predicate residual is suggested for these drill moment components. 


\subsection{Zero transverse normal stress assumption}

The transverse normal force $\bar{m}_{0}^{33}:=\overline{\mathbf{g}}^{3} \cdot \mathbf{m}_{0}^{3}=\mathbf{a}^{3} \cdot \mathbf{m}_{0}^{3}=m_{0}^{33}$ does not enter the shell balance equations, neither in $(105,106)$ nor in $(139)-(142)$. Consequently, there is a freedom to make the assumption that

$$
m_{0}^{33}=\frac{1}{\sqrt{a}} \int_{\zeta^{-}}^{\zeta^{+}} \sqrt{g} \sigma^{33} \mathrm{~d} \xi^{3} \stackrel{!}{=} 0 .
$$

Since all higher-order moments $\mathbf{m}_{1}^{3}, \mathbf{m}_{2}^{3}, \ldots$ vanish according to (87.3) and (86.1), Eq. (152) is equivalent to

$$
\sigma^{33}\left(\xi^{\alpha}, \xi^{3}\right) \equiv 0,
$$

which justifies to call (152) the zero transverse normal stress assumption. It is inevitably linked with an additional assumption,

$$
p_{1}^{3}=\frac{1}{\sqrt{a}} \int_{\zeta^{-}}^{\zeta^{+}} \sqrt{g} \xi f^{3} \mathrm{~d} \xi+\frac{1}{\sqrt{a}}\left[\sqrt{g} \xi t^{3}\right]_{\xi^{-}}^{\xi^{+}} \stackrel{!}{=} 0,
$$

claiming that there is no distributed through-the-thickness loading. Note that $p_{1}^{3}$ and the drill component $l^{3}$ are entirely unrelated to each other.

In this context, conventional treatments of shell theory adopt the following argumentation: Condition (152) can be applied to eliminate the degree of freedom related to the thickness change from the constitutive law via static condensation. This leads to a freedom in choosing the length of the convected basis vector $\mathbf{g}_{3}$. For the sake of convenience, one chooses $\left\|\mathbf{g}_{3}\right\|=1$ in accordance with the Reissner-Mindlin assumption.

Here, the Reissner-Mindlin assumption (or another kinematic restriction) is not applied, which has already been emphasized. Instead, the additional static assumptions (152) and (154) can be used to simplify the moment balance (147): The balance Eq. (112) reads in the present context, see (15.3) and (128.1),

$$
b_{\beta \alpha} m^{\beta \alpha}=\lambda\left(m_{0}^{33}-p_{1}^{3}\right) .
$$

Consequently, assuming (152) and (154), the term $b_{\beta \alpha} m^{\beta \alpha}$ in (145.1) vanishes,

$$
\left.m^{\beta \alpha}\right|_{\alpha}+\gamma^{\mu} b_{\mu \alpha} m^{\beta \alpha}-m_{0}^{3 \beta}+l^{\beta}=0 .
$$

At first glance, one could be misled to identify $m_{0}^{3 \beta}-\gamma^{\mu} b_{\mu \alpha} m_{1}^{\beta \alpha}$ with $m_{0}^{\beta 3}$, in analogy with (110.1). However, relation (110.1) only holds for the components with respect to the convected frame; see Sect. 5.1.

\subsection{The role of thinness}

For sufficiently thin shells, it makes sense to postulate

$$
\left|\gamma^{\mu} b_{\mu \alpha} m^{\beta \alpha}\right| \ll\left|m_{0}^{3 \beta}\right|
$$

such that the moment balance Eq. (156) finally turns into (147). Roughly speaking, condition (157) is feasible as long as bending moments $m^{\beta \alpha}$ are 'small'. A more rigorous justification for (157) is obtained as follows. For this purpose it is convenient to refer all relevant vector and tensor components to a normalized principal axes frame of the curvature tensor $b_{\alpha}^{\mu}$ and to make use of the principal curvatures $R_{1}$ and $R_{2}$. Then,

$$
\gamma^{\mu} b_{\mu \alpha} m^{\beta \alpha}=\frac{\gamma_{1}}{R_{1}} m^{\beta 1}+\frac{\gamma_{2}}{R_{2}} m^{\beta 2} .
$$

Common dimensionality considerations lead to the estimates for shear and bending strains (defined in Sect. 6),

$$
\gamma_{\beta}=O(1), \quad \varepsilon_{\beta \alpha}^{(1)}=O\left(\frac{1}{t}\right) .
$$

Correspondingly, for shear forces and bending moments,

$$
m_{0}^{3 \beta}=O(t), \quad m^{\beta \alpha}=O\left(t^{2}\right) .
$$


Inserting these estimates in (158) yields

$$
\left|\gamma^{\mu} b_{\mu \alpha} m^{\beta \alpha}\right| \lesssim \frac{t}{R}\left|m_{0}^{3 \beta}\right|
$$

with $R=\min \left\{\left|R_{1}\right|,\left|R_{2}\right|\right\}$ being the minimum curvature radius. That is, as long as the assumptions leading to the estimates (160) hold, condition (157) is a consequence of thinness, i.e., of

$$
\frac{t}{R} \ll 1
$$

\section{Recovery of the geometrically exact shell theory}

\subsection{The kinematic variables and the principle of virtual displacements}

To complete the derivation of the first-order approximation, the kinematic variables are again derived via the principle of virtual displacements. For the hierarchical theory, the PVD has been given in Sect. 3.1. Its first-order counterpart is obtained applying the static constraints (86),

$$
\begin{aligned}
& \int_{\mathcal{A}}\left(\mathbf{m}_{0}^{\alpha} \cdot \delta \mathbf{u}_{0, \alpha}+\mathbf{m}_{0}^{3} \cdot \delta \mathbf{u}_{1}+\mathbf{m}_{1}^{\alpha} \cdot \delta \mathbf{u}_{1, \alpha}+2 \mathbf{m}_{1}^{3} \cdot \delta \mathbf{u}_{2}\right) \mathrm{d} A \\
& =\int_{\mathcal{A}}\left(\mathbf{p}_{0} \cdot \delta \mathbf{u}_{0}+\mathbf{p}_{1} \cdot \delta \mathbf{u}_{1}\right) \mathrm{d} A+\int_{\partial \mathcal{A}}\left(\mathbf{M}_{0} \cdot \delta \mathbf{u}_{0}+\mathbf{M}_{1} \cdot \delta \mathbf{u}_{1}\right) \mathrm{d} s .
\end{aligned}
$$

The fourth term on the left-hand side is the only one which contains the second-order virtual displacement vector $\delta \mathbf{u}_{2}$. It thus leads immediately to the trivial balance equation $\mathbf{m}_{1}^{3}=\mathbf{0}$ and will therefore be omitted. Making use of the pseudo-force vector $\mathbf{n}^{\alpha}=\mathbf{m}_{0}^{\alpha}-m_{1}^{\beta \mu} \mathbf{g}_{3, \mu}^{c}$, introduced in (104), the virtual strain energy density, i.e., the integrand on the left-hand side, becomes

$$
\begin{aligned}
\delta w^{\prime}= & \left(n^{\beta \alpha} \mathbf{a}_{\beta}+n^{3 \alpha} \overline{\mathbf{g}}_{3}^{c}+m_{1}^{\alpha \mu} \overline{\mathbf{g}}_{3, \mu}^{c}\right) \cdot \delta \mathbf{u}_{0, \alpha}+n^{\alpha 3} \mathbf{a}_{\alpha} \cdot \delta \mathbf{u}_{1} \\
& +m_{0}^{33} \overline{\mathbf{g}}_{3}^{c} \cdot \delta \mathbf{u}_{1}+m_{1}^{\beta \alpha} \mathbf{a}_{\beta} \cdot \delta \mathbf{u}_{1, \alpha} \\
= & n^{\beta \alpha} \mathbf{a}_{\beta} \cdot \delta \mathbf{a}_{\alpha}+n^{3 \alpha}\left(\overline{\mathbf{g}}_{3}^{c} \cdot \delta \mathbf{a}_{\alpha}+\mathbf{a}_{\alpha} \cdot \delta \overline{\mathbf{g}}_{3}^{c}\right)+ \\
& +m_{0}^{33} \overline{\mathbf{g}}_{3}^{c} \cdot \delta \overline{\mathbf{g}}_{3}^{c}+m_{1}^{\beta \alpha}\left(\mathbf{a}_{\beta} \cdot \delta \overline{\mathbf{g}}_{3, \alpha}^{c}+\overline{\mathbf{g}}_{3, \alpha}^{c} \cdot \delta \mathbf{a}_{\beta}\right) .
\end{aligned}
$$

Note that all tensor components refer to the convected frame (the left-hand superscript 'c' has again been omitted for notational convenience). For the second equality, use has been made of the symmetry relation (103) and of

$$
\delta \mathbf{u}_{0, \alpha}=\delta \mathbf{a}_{\alpha}, \quad \delta \mathbf{u}_{1}=\delta \mathbf{x}_{1}=\delta \overline{\mathbf{g}}_{3}^{c} .
$$

Respecting the symmetry of pseudo-forces $n^{\beta \alpha}$, expression (164) gives rise to the definition of the strain measures

$$
\left.\begin{array}{rl}
\delta \varepsilon_{\beta \alpha}^{(0)} & =\frac{1}{2}\left(\mathbf{a}_{\beta} \cdot \delta \mathbf{a}_{\alpha}+\mathbf{a}_{\alpha} \cdot \delta \mathbf{a}_{\beta}\right) \\
\delta \varepsilon_{\beta \alpha}^{(1)} & =\mathbf{a}_{\beta} \cdot \delta \overline{\mathbf{g}}_{3, \alpha}^{c}+\overline{\mathbf{g}}_{3, \alpha}^{c} \cdot \delta \mathbf{a}_{\beta} \\
\delta \varepsilon_{3 \alpha}^{(0)} & =\frac{1}{2}\left(\overline{\mathbf{g}}_{3}^{c} \cdot \delta \mathbf{a}_{\alpha}+\mathbf{a}_{\alpha} \cdot \delta \overline{\mathbf{g}}_{3}^{c}\right) \\
\delta \varepsilon_{33}^{(0)} & =\overline{\mathbf{g}}_{3}^{c} \cdot \delta \overline{\mathbf{g}}_{3}^{c}
\end{array}\right\},
$$

and the strain energy density (164) finally reads

$$
\delta w^{\prime}=n^{\beta \alpha} \delta \varepsilon_{\beta \alpha}^{(0)}+m_{1}^{\beta \alpha} \delta \varepsilon_{\beta \alpha}^{(1)}+n^{3 \alpha} 2 \delta \varepsilon_{3 \alpha}^{(0)}+m_{0}^{33} \delta \bar{\varepsilon}_{33}^{(0)} .
$$


The virtual strain measures can be considered as the variations of their physical counterparts,

$$
\left.\begin{array}{rl}
\varepsilon_{\beta \alpha}^{(0)} & =\frac{1}{2}\left(\mathbf{a}_{\beta} \cdot \mathbf{a}_{\alpha}-\mathbf{A}_{\beta} \cdot \mathbf{A}_{\alpha}\right)=\frac{1}{2}\left(a_{\beta \alpha}-A_{\beta \alpha}\right) \\
\varepsilon_{\beta \alpha}^{(1)} & =\mathbf{a}_{\beta} \cdot \overline{\mathbf{g}}_{3, \alpha}^{c}-\mathbf{A}_{\beta} \cdot \mathbf{A}_{3, \alpha}=\left.\left(\lambda \gamma_{\beta}\right)\right|_{\alpha}-\lambda b_{\beta \alpha}+B_{\beta \alpha} \\
\varepsilon_{3 \alpha}^{(0)} & =\frac{1}{2}(\overline{\mathbf{g}}_{3}^{c} \cdot \mathbf{a}_{\alpha}-\underbrace{\mathbf{A}_{3} \cdot \mathbf{A}_{\alpha}}_{=0})=\frac{1}{2} \lambda \gamma_{\alpha} \\
\varepsilon_{33}^{(0)} & =\frac{1}{2}(\overline{\mathbf{g}}_{3}^{c} \cdot \overline{\mathbf{g}}_{3}^{c}-\underbrace{\mathbf{A}_{3} \cdot \mathbf{A}_{3}}_{=1})=\frac{1}{2}\left(\lambda^{2}-1+\lambda^{2} \gamma_{\alpha} \gamma^{\alpha}\right)
\end{array}\right\} .
$$

Recall that (167) has been derived here via the hierarchical approach under the restriction of the statical assumptions (86). In contrast, the 'conventional approach' is as follows: Adopting the kinematic assumptions

$$
\mathbf{g}_{\alpha}^{c}\left(\xi^{\beta}, \xi^{3}\right)=\mathbf{a}_{\alpha}\left(\xi^{\beta}\right)+\xi^{3} \overline{\mathbf{g}}_{3, \alpha}^{c}\left(\xi^{\beta}\right), \quad \mathbf{g}_{3}^{c}\left(\xi^{\beta}, \xi^{3}\right) \equiv \overline{\mathbf{g}}_{3}^{c}\left(\xi^{\beta}\right)
$$

in the expression for the virtual strain energy,

$$
\delta W_{\text {int }}=\int_{\mathcal{B}} \mathbf{P}: \delta \mathbf{F} \mathrm{d} V_{0}=\int_{\mathcal{B}} \mathbf{s}^{i} \cdot \delta \mathbf{g}_{i}^{c} \mathrm{~d} V=\int_{\mathcal{A}} \delta w^{\prime} \mathrm{d} A,
$$

a similar expression for the virtual strain energy density is obtained [33],

$$
\delta w^{\prime \prime}=n^{\beta \alpha} \delta \varepsilon_{\beta \alpha}^{(0)}+m_{1}^{\beta \alpha} \delta \varepsilon_{\beta \alpha}^{(1)}+n^{3 \alpha} 2 \delta \varepsilon_{3 \alpha}^{(0)}+m_{1}^{3 \alpha} 2 \delta \varepsilon_{3 \alpha}^{(1)}+\tilde{m}_{0}^{33} \delta \bar{\varepsilon}_{33}^{(0)},
$$

which involves the components of the pseudo transverse force vector

$$
\tilde{\mathbf{m}}_{0}^{3}:=\mathbf{m}_{0}^{3}-m_{1}^{3 \mu} \overline{\mathbf{g}}_{3, \mu}=\tilde{m}_{0}^{\beta 3} \mathbf{a}_{\beta}+\tilde{m}_{0}^{33} \overline{\mathbf{g}}_{3}^{c}
$$

and additional virtual strain variables

$$
\delta \varepsilon_{3 \alpha}^{(1)}=\frac{1}{2}\left(\overline{\mathbf{g}}_{3}^{c} \cdot \delta \overline{\mathbf{g}}_{3, \alpha}^{c}+\overline{\mathbf{g}}_{3, \alpha}^{c} \cdot \delta \overline{\mathbf{g}}_{3}^{c}\right)
$$

deriving from

$$
\varepsilon_{3 \alpha}^{(1)}=\frac{1}{2}(\overline{\mathbf{g}}_{3}^{c} \cdot \overline{\mathbf{g}}_{3, \alpha}^{c}-\underbrace{\mathbf{A}_{3} \cdot \mathbf{A}_{3, \alpha}}_{=0}) .
$$

Due to moment balance, $\tilde{m}_{0}^{\beta 3}=n^{3 \beta}$. Note that the $\varepsilon_{3 \alpha}^{(1)}$ are not independent variables. Instead, $\varepsilon_{3 \alpha}^{(1)}=\varepsilon_{33, \alpha}^{(0)}$. The strain energy expressions of the hierarchic and of the conventional approach, (167) and (171), respectively, coincide if the static assumptions of the former are supplemented by the zero transverse normal stress assumption (152),

$$
m_{0}^{33} \stackrel{!}{=} 0
$$

on the one hand, and the kinematic assumptions of the latter by the transverse non-extensibility constraint

$$
\varepsilon_{33}^{(0)} \stackrel{!}{=} 0
$$

on the other. Consequently, the common expression

$$
\delta w=n^{\beta \alpha} \delta \varepsilon_{\beta \alpha}^{(0)}+m_{1}^{\beta \alpha} \delta \varepsilon_{\beta \alpha}^{(1)}+n^{3 \alpha} 2 \delta \varepsilon_{3 \alpha}^{(0)}
$$

is obtained. That is, the two conceptionally totally different approaches deliver the same expression for the PVD. To be more precise, it is mentioned that the two resulting theories still differ with respect to one aspect. The hierarchic approach yields the symmetry of bending moments, $m_{1}^{\beta \alpha}=m_{1}^{\alpha \beta}$, and the absence of shear force couples, $m_{1}^{3 \alpha}=0$, as additional balance equations. In contrast, these conditions are merely postulated in the context of the conventional approach. 


\subsection{Elimination of thickness stretch}

For the conventional approach, the degree of freedom related to the thickness stretch is explicitly omitted due to the condition (175). This is not the case for the hierarchic approach where a through-the-thickness straining is present implicitly and is represented by the variable $\lambda$. In principle, $\lambda$ could be determined via the constitutive relation from the zero transverse normal stress assumption and thereby eliminated from the formulation. A more feasible approach is described here. Given the assumptions (compare Sect. 5.4)

$$
m_{0}^{33} \stackrel{!}{=} 0, \quad \bar{p}_{1}^{3}=0, \quad \bar{M}_{1}^{3}=0
$$

the variable $\lambda$ can be eliminated formally from the PVD without referring to a specific constitutive relation. Note that the external moments $\bar{p}_{1}^{3}$ and $\bar{M}_{1}^{3}$ are related to a through-the-thickness loading, compare (58) and (57). They must not be confused with the external drill moment components $l^{3}$ or $L^{3}$. To proceed, the derivations of the previous Section are recapitulated while decomposing force and moment vectors with respect to the skew director frame $\left(\mathbf{a}_{\beta}, \overline{\mathbf{g}}_{3}\right)$. By this means, the thickness stretch variable $\lambda$ explicitly enters the PVD. In the following, the component notation introduced in Sect. 5.2 is adopted. That is, bared variables refer to the skew director frame and non-bared ones to the surface orthogonal frame. According to $\overline{\mathbf{g}}_{3}^{c}=\lambda \overline{\mathbf{g}}_{3}$,

$$
\begin{aligned}
& \delta \mathbf{u}_{1}=\overline{\mathbf{g}}_{3} \delta \lambda+\lambda \delta \overline{\mathbf{g}}_{3}, \\
& \delta \mathbf{u}_{1, \alpha}=\overline{\mathbf{g}}_{3, \alpha} \delta \lambda+\overline{\mathbf{g}}_{3} \delta \lambda_{, \alpha}+\lambda_{, \alpha} \delta \overline{\mathbf{g}}_{3}+\lambda \delta \overline{\mathbf{g}}_{3, \alpha} .
\end{aligned}
$$

Converting the term with the $\delta \lambda_{, \alpha}$ via the product rule and the divergence theorem, considering that $\bar{m}_{1}^{3 \alpha}=$ $\bar{m}_{1}^{\beta 3}=\bar{m}_{1}^{33}=0$, and arranging the virtual work terms in a convenient way, (163) is reformulated equivalently,

$$
\begin{aligned}
& \int_{\mathcal{A}}\left\{\left(\bar{m}_{0}^{\beta \alpha}-\gamma_{3 \mu}^{\beta} \bar{m}^{\alpha \mu}\right) \mathbf{a}_{\beta} \cdot \delta \mathbf{a}_{\alpha}+\left(\bar{m}_{0}^{3 \alpha}-\gamma_{3 \mu}^{3} \bar{m}^{\alpha \mu}\right) \overline{\mathbf{g}}_{3} \cdot \delta \mathbf{a}_{\alpha}\right. \\
& +\left(\lambda \bar{m}_{0}^{\beta 3}+\frac{\lambda, \mu}{\lambda} \bar{m}^{\beta \mu}\right) \mathbf{a}_{\beta} \cdot \delta \overline{\mathbf{g}}_{3}+\bar{m}^{\beta \alpha}\left(\mathbf{a}_{\beta} \cdot \delta \overline{\mathbf{g}}_{3, \alpha}+\overline{\mathbf{g}}_{3, \alpha} \cdot \delta \mathbf{a}_{\beta}\right)+\lambda \bar{m}_{0}^{33} \overline{\mathbf{g}}_{3} \cdot \delta \overline{\mathbf{g}}_{3} \\
& +\left[\underline{\bar{m}_{0}^{33}-\gamma_{\beta \alpha}^{3} \bar{m}_{1}^{\beta \alpha}-\bar{p}_{1}^{3}}+\gamma_{\beta}\left(\underline{\left.\bar{m}_{0}^{\beta 3}-\bar{m}_{1}^{\beta \alpha} \|_{\alpha}-\bar{p}_{1}^{\beta}\right)}+\gamma_{\beta} \gamma^{\beta}\left(\bar{m}_{0}^{33}-\bar{p}_{1}^{3}\right)\right] \delta \lambda\right\} \mathrm{d} A \\
& =\int_{\mathcal{A}}\left(\mathbf{p}_{0} \cdot \delta \mathbf{u}_{0}+\lambda \mathbf{p}_{1} \cdot \delta \overline{\mathbf{g}}_{3}\right) \mathrm{d} A+\int_{\partial \mathcal{A}}\left(\mathbf{M}_{0} \cdot \delta \mathbf{u}_{0}+\lambda \mathbf{M}_{1} \cdot \delta \overline{\mathbf{g}}_{3}\right) \mathrm{d} s .
\end{aligned}
$$

The two underlined expressions in the third line vanish at equilibrium due to (112) and (108.1), respectively. The other terms involving $\bar{m}_{0}^{33}, \bar{p}_{1}^{3}$, and $\bar{M}_{1}^{3}$ disappear as a consequence of assumptions (177). Considering further (134) as well as $\bar{l}^{\beta}=\lambda \bar{p}_{1}^{\beta}$ and, correspondingly, $\bar{L}^{\beta}=\lambda \bar{M}_{1}^{\beta}$, the thickness stretch $\lambda$ does not show up in the PVD anymore,

$$
\begin{gathered}
\int_{\mathcal{A}}\left[\bar{n}^{\beta \alpha} \mathbf{a}_{\beta} \cdot \delta \mathbf{a}_{\alpha}+\bar{n}^{3 \alpha}\left(\overline{\mathbf{g}}_{3} \cdot \delta \mathbf{a}_{\alpha}+\mathbf{a}_{\beta} \cdot \delta \overline{\mathbf{g}}_{3}\right)+\bar{m}^{\beta \alpha}\left(\mathbf{a}_{\beta} \cdot \delta \overline{\mathbf{g}}_{3, \alpha}+\overline{\mathbf{g}}_{3, \alpha} \cdot \delta \mathbf{a}_{\alpha}\right)\right] \mathrm{d} A \\
=\int_{\mathcal{A}}\left(\mathbf{p}_{0} \cdot \delta \mathbf{u}_{0}+\bar{l}^{\beta} \mathbf{a}_{\beta} \cdot \delta \overline{\mathbf{g}}_{3}\right) \mathrm{d} A+\int_{\partial \mathcal{A}}\left(\mathbf{M}_{0} \cdot \delta \mathbf{u}_{0}+\bar{L}^{\beta} \mathbf{a}_{\beta} \cdot \delta \overline{\mathbf{g}}_{3}\right) \mathrm{d} s .
\end{gathered}
$$

In the present context, pseudo-membrane and pseudo-shear forces read

$$
\begin{aligned}
& \bar{n}^{\beta \alpha}:=\bar{m}_{0}^{\beta \alpha}-\gamma_{3 \mu}^{\beta} \bar{m}^{\alpha \mu}, \\
& \bar{n}^{3 \alpha}:=\bar{m}_{0}^{3 \alpha}-\gamma_{3 \mu}^{3} \bar{m}^{\alpha \mu}=\lambda \bar{m}_{0}^{\beta 3}+\frac{\lambda, \mu}{\lambda} \bar{m}^{\beta \mu} .
\end{aligned}
$$

Considering the symmetry of the $\bar{n}^{\beta \alpha}$, the virtual strain variables entering (181) are

$$
\left.\begin{array}{rl}
\delta \bar{\varepsilon}_{\beta \alpha}^{(0)} & =\frac{1}{2}\left(\mathbf{a}_{\beta} \cdot \delta \mathbf{a}_{\alpha}+\mathbf{a}_{\alpha} \cdot \delta \mathbf{a}_{\beta}\right) \\
\delta \bar{\varepsilon}_{\beta \alpha}^{(1)} & =\mathbf{a}_{\beta} \cdot \delta \overline{\mathbf{g}}_{3, \alpha}+\overline{\mathbf{g}}_{3, \alpha} \cdot \delta \mathbf{a}_{\beta} \\
\delta \bar{\varepsilon}_{3 \alpha}^{(0)} & =\frac{1}{2}\left(\overline{\mathbf{g}}_{3} \cdot \delta \mathbf{a}_{\alpha}+\mathbf{a}_{\alpha} \cdot \delta \overline{\mathbf{g}}_{3}\right)
\end{array}\right\}
$$


such that the virtual strain energy expression still formally coincides with its original appearance (176),

$$
\delta w=\bar{n}^{\beta \alpha} \delta \bar{\varepsilon}_{\beta \alpha}^{(0)}+\bar{m}^{\beta \alpha} \delta \bar{\varepsilon}_{\beta \alpha}^{(1)}+n^{3 \alpha} 2 \delta \bar{\varepsilon}_{3 \alpha}^{(0)} .
$$

The physical counterparts of virtual strains (184) read

$$
\left.\begin{array}{l}
\bar{\varepsilon}_{\beta \alpha}^{(0)}=\frac{1}{2}\left(\mathbf{a}_{\beta} \cdot \mathbf{a}_{\alpha}-\mathbf{A}_{\beta} \cdot \mathbf{A}_{\alpha}\right)=\frac{1}{2}\left(a_{\beta \alpha}-A_{\beta \alpha}\right) \\
\bar{\varepsilon}_{\beta \alpha}^{(1)}=\mathbf{a}_{\beta} \cdot \overline{\mathbf{g}}_{3, \alpha}-\mathbf{A}_{\beta} \cdot \mathbf{A}_{3, \alpha}=\left.\gamma_{\beta}\right|_{\alpha}-b_{\beta \alpha}+B_{\beta \alpha} \\
\bar{\varepsilon}_{3 \alpha}^{(0)}=\frac{1}{2}\left(\overline{\mathbf{g}}_{3} \cdot \mathbf{a}_{\alpha}-\mathbf{A}_{3} \cdot \mathbf{A}_{\alpha}\right)=\frac{1}{2} \gamma_{\alpha}
\end{array}\right\} .
$$

Concluding, neither the kinematic relations nor the PVD are affected formally by a scaling $\frac{1}{\lambda}$ of the director vector $\overline{\mathbf{g}}_{3}=\frac{1}{\lambda} \overline{\mathbf{g}}_{3}^{c}$. This conclusion holds under the assumptions (177). Note that no additional approximations are involved in the derivation. Consequently, for any choice of this scaling, the same structure of the theory is obtained. In particular, the scaling can be chosen such that $\left\|\overline{\mathbf{g}}_{3}\right\|=1$ : Replacing $\lambda$ by a $\lambda^{\prime}=\lambda \sqrt{1+\gamma_{\beta} \gamma^{\beta}}$, the previous derivations apply analogously. In this way, conventional Reissner-Mindlin kinematics are recovered. This choice of scaling is of exceptional practical relevance since it allows to parameterize the kinematics of the director vector by means of rotational degrees of freedom [31].

Remark Certainly, any kinematic variable can be eliminated from the PVD with the help of the corresponding balance equation. What has been shown here is that the variable $\lambda$ can be eliminated without changing the formal structure of the theory.

\section{Conclusions}

To summarize, an infinite hierarchy of nonlinear $\ell$ th-order shell theories has been derived from threedimensional continuum mechanics based on a polynomial series expansion of the displacement field. Posing a static assumption, namely that second- and higher-order moments vanish, a first-order approximation is obtained which can be considered as a 'conventional' shell theory. In particular, it is shown that, within the same theoretical framework, the static assumption on the one hand and the common Reisner-Mindlin-type kinematic assumption on the other lead to the same theory, here called the 'geometrically exact' shell theory. This coincidence, which is well known from the linear theory, is explicated in full generality. As a side result, the unavoidable appearance of transverse $(=$ drill $)$ moment components is analysed. Feasible approximations are formulated which allow to separate these drill moment components from the remaining balance equations without affecting the equilibrium of the standard static variables (membrane and shear forces, bending moments). Interestingly, these approximations are well known from conventional treatments of shell theory, although they appear in a different context there. This procedure leads to a favourable structure of the component representation of balance equations in the sense that they formally coincide with the ones of linear shear-deformable shell theory. This is worth to be mentioned since former formulations of the geometrically exact theory come along with component equations which are hardly manageable due to the explicit appearance of Christoffel symbols. Most of these results are of rather theoretical interest. They help to better understand the relation of different shell theories and to integrate these in a common framework. The following aspects shall be emphasized:

(i) Introducing a spatial (i.e., length measuring with respect to the deformed configuration) through-thethickness coordinate, the 'skew director' coordinate system emerges in a natural way and turns out be particularly beneficial to analyse the exact role of shear deformation and thickness stretch in the context of the first-order approximation.

(ii) To correctly classify the role of transverse (= drill) moment components, it is differentiated between the 'genuine' drill moments of the statically exact theory and 'residual' ones. Eliminating the latter, the component representation of the shell balance equations turn into a particularly beneficial format.

(iii) The residual drill moments need not to be in equilibrium with the external moments. That is, no external transverse moment components are required to equilibrate the internal ones. Consequently, external moments can be applied tangent to the reference surface disregarding any possible shear deformation. Although this might appear as a matter of course, it is not consistent with the geometrically exact theory a priori. 
(iv) It is clearly separated between a 'hierarchic' approach applying static assumptions on the one hand and the 'conventional' approach based on kinematic assumptions on the other.

(v) In the conventional approach, the thickness stretch variable is explicitly excluded from the formulation via a kinematic assumption. In contrast, for the hierarchic approach, the zero transverse normal stress assumption can be used to eliminate the thickness stretch variable from the PVD without altering its formal structure.

(vi) The membrane theory is commonly derived as a special case of conventional shell theory, either of Kirchhoff-Love or of Reissner-Mindlin type. It is an interesting side result that the membrane theory derives from the hierarchic approach with one single assumption, i.e., the absence of bending moments, disregarding any additional kinematic or static constraints (concerning the cross-sectional deformation or higher-order [= warping] moments).

Here, the hierarchic point of view is clearly propagated since its basis, the static assumptions, have a very natural appearance. This is in contrast to the Reissner-Mindlin kinematic assumption, which is of rather heuristic nature. This conception is supported by two observations: Firstly, if Reissner-Mindlin kinematics would reflect a physical deformation, any transverse shear deformation would come along with a contraction in the thickness direction. This clearly disagrees with the empirical perception and is the reason why ReissnerMindlin-type theories are not asymptotically consistent with three-dimensional continuum mechanics [22,27]. ${ }^{4}$ Secondly, from a more practical point of view, it turns out that, whenever higher-order approaches become relevant, such as for sandwich shell structures, warping moments due to a constraining of cross-sectional warping have more influence on the overall behaviour of the structure than the effect of cross-sectional warping (due to self-equilibration of internal forces) itself. ${ }^{5}$ From this it can be concluded that, when choosing an appropriate level of approximation, the primary question is, which maximum order of stress resultants is required. The corresponding kinematics then follow from considerations on the asymptotic consistency.

So far, in the context of nonlinear shell theories, the Reissner-Mindlin assumption has been justified $a$ posteriori in the sense that its implementations have proven to deliver feasible results. The present hierarchic approach can be considered as a step further since it provides a theoretical justification as well. From the hierarchic point of view, the picture is as follows: Cross-sectional and through-the thickness deformations are considered to be unconstrained a priori. Due to self-equilibration of the corresponding static variables, crosssectional warping and thickness straining appear such that higher-order moments as well as the transverse normal force (almost) vanish. As long as these static variables are in self-equilibrium, the corresponding kinematic variables can be eliminated from the formulation such that only the standard variables remain. From this point of view, Reissner-Mindlin kinematics are applied to reproduce the relevant part of shell deformation but not to constrain the shell deformation. This conception is supported by the observation that ReissnerMindlin-type shell theories are able to deliver feasible results (related to deformation and in-plane stresses) even for weak-core sandwich structures, e.g., where relevant cross-sectional warping actually occurs. ${ }^{6}$

Acknowledgements Open access funding provided by University of Innsbruck and Medical University of Innsbruck.

Open Access This article is distributed under the terms of the Creative Commons Attribution 4.0 International License (http:// creativecommons.org/licenses/by/4.0/), which permits unrestricted use, distribution, and reproduction in any medium, provided you give appropriate credit to the original author(s) and the source, provide a link to the Creative Commons license, and indicate if changes were made.

\section{A Mathematical prerequisites}

A.1 Some vector and tensor algebra

It is convenient to introduce the Kronecker symbol

$$
\delta_{j}^{i}= \begin{cases}1, & i=j \\ 0, & i \neq j\end{cases}
$$

\footnotetext{
${ }^{4}$ That is, Reissner-Mindlin theories on the one hand and three-dimensional nonpolar continuum mechanics on the other do not lead to the same results in the limit $t \rightarrow 0$.

5 This effect is more commonly known from the torsion of rods with constrained warping.

${ }^{6}$ In accordance with what has been mentioned in the previous Paragraph, this is true as long as no constraining of the crosssectional deformation is present.
} 
and the permutation symbol

$$
\epsilon(i, j, k)=\left\{\begin{array}{ll}
1, & (i, j, k) \text { is an even permutation of }(1,2,3) \\
0, & (i, j, k) \text { is not a permutation of }(1,2,3) \\
-1, & (i, j, k) \text { is an odd permutation of }(1,2,3)
\end{array} .\right.
$$

To proceed, a curvilinear coordinate system with basis vectors $\left(\mathbf{g}_{1}, \mathbf{g}_{2}, \mathbf{g}_{3}\right)$ is embedded in the three-dimensional Euclidean space. The basis vectors span a parallelepiped with the volume

$$
\sqrt{g}=\left(\mathbf{g}_{1} \times \mathbf{g}_{2}\right) \cdot \mathbf{g}_{3} \text {. }
$$

The components of the totally antisymmetric epsilon tensor,

$$
\epsilon_{i j k}=\sqrt{g} \epsilon(i, j, k), \quad \epsilon^{i j k}=\frac{1}{\sqrt{g}} \epsilon(i, j, k)
$$

can be used to relate co- and contravariant basis vectors,

$$
\mathbf{g}^{i}=\frac{1}{2} \epsilon^{i j k} \mathbf{g}_{j} \times \mathbf{g}_{k}, \quad \mathbf{g}_{i}=\frac{1}{2} \epsilon_{i j k} \mathbf{g}^{j} \times \mathbf{g}^{k}
$$

and

$$
\mathbf{g}_{i} \times \mathbf{g}_{j}=\epsilon_{i j k} \mathbf{g}^{k}, \quad \mathbf{g}^{i} \times \mathbf{g}^{j}=\epsilon^{i j k} \mathbf{g}_{k} .
$$

Analogously, for a surface with the basis vectors $\left(\mathbf{a}_{1}, \mathbf{a}_{2}\right)$ embedded in the three-dimensional Euclidean space, the antisymmetric surface epsilon tensor is

$$
\epsilon_{\alpha \beta}=\sqrt{a} \epsilon(\alpha, \beta, 3), \quad \epsilon^{\alpha \beta}=\frac{1}{\sqrt{a}} \epsilon(\alpha, \beta, 3)
$$

with

$$
\sqrt{a}=\left\|\mathbf{a}_{1} \times \mathbf{a}_{2}\right\|
$$

being the area of the parallelogram spanned by the basis vectors. Making use of the surface unit normal vector

$$
\mathbf{a}_{3}=\frac{\mathbf{a}_{1} \times \mathbf{a}_{2}}{\sqrt{a}}
$$

the contravariant basis vectors can be given as

$$
\mathbf{a}^{\alpha}=\epsilon^{\alpha \beta} \mathbf{a}_{\beta} \times \mathbf{a}_{3}, \quad \mathbf{a}^{3}=\frac{1}{2} \epsilon^{\alpha \beta} \mathbf{a}_{\alpha} \times \mathbf{a}_{\beta}=\mathbf{a}_{3} .
$$

Conversely,

$$
\mathbf{a}_{\alpha}=\epsilon_{\alpha \beta} \mathbf{a}^{\beta} \times \mathbf{a}^{3}, \quad \mathbf{a}_{3}=\frac{1}{2} \epsilon_{\alpha \beta} \mathbf{a}^{\alpha} \times \mathbf{a}^{\beta}
$$

and

$$
\mathbf{a}_{3} \times \mathbf{a}_{\alpha}=\epsilon_{\alpha \beta} \mathbf{a}^{\beta}, \quad \mathbf{a}_{\alpha} \times \mathbf{a}_{\beta}=\epsilon_{\alpha \beta} \mathbf{a}^{3} .
$$

Further helpful relations are

$$
\epsilon^{\alpha \mu} \epsilon_{\beta \nu}=\delta_{\beta}^{\alpha} \delta_{v}^{\mu}-\delta_{v}^{\alpha} \delta_{\beta}^{\mu}, \quad \epsilon^{\alpha \mu} \epsilon_{\beta \mu}=\delta_{\beta}^{\alpha}, \quad \epsilon^{\alpha \mu} \epsilon_{\alpha \mu}=\delta_{\alpha}^{\alpha}=2 .
$$

The covariant, contravariant, and mixed metric coefficients of the surface metric tensor

$$
a_{\alpha \beta}=\mathbf{a}_{\alpha} \cdot \mathbf{a}_{\beta}, \quad a^{\alpha \beta}=\mathbf{a}^{\alpha} \cdot \mathbf{a}^{\beta}, \quad \delta_{\beta}^{\alpha}=\mathbf{a}^{\alpha} \cdot \mathbf{a}_{\beta}
$$

can be used to lower, raise, and to exchange indices, respectively. That is, for any surface vector $\mathbf{y}=y^{\alpha} \mathbf{a}_{\alpha}=$ $y_{\alpha} \mathbf{a}^{\alpha}$,

$$
y_{\alpha}=a_{\alpha \mu} y^{\mu}, \quad y^{\alpha}=a^{\alpha \mu} y_{\mu}, \quad y^{\alpha}=\delta_{\mu}^{\alpha} y^{\mu} .
$$

In particular, $a^{\alpha \mu} a_{\mu \beta}=\delta_{\beta}^{\alpha}$. Finally, due to

$$
2=\epsilon^{\alpha \mu} \epsilon_{\alpha \mu}=\epsilon^{\alpha \mu} \epsilon^{\beta \nu} a_{\alpha \beta} a_{\mu \nu}=\frac{2}{a}\left(a_{11} a_{22}-a_{12} a_{21}\right)=2 \frac{\left|a_{\alpha \beta}\right|}{a}
$$

the square $a$ of so-called Gram's determinant $\sqrt{a}$ turns out to equal the determinant of the covariant metric coefficient matrix. 
A.2 Basic tensor analysis on a surface

A shell reference surface $\mathcal{A}$ shall be spanned by position vectors $\overline{\mathbf{x}}=\overline{\mathbf{x}}\left(\xi^{\alpha}\right)$. The parameters $\xi^{\alpha}, \alpha=1,2$, are the (curvilinear) coordinates of the surface. Here, they are considered to be dimensionless. A natural choice for the covariant basis vectors is then

$$
\mathbf{a}_{\alpha}=\overline{\mathbf{x}}_{, \alpha} \equiv \frac{\partial \overline{\mathbf{x}}}{\partial \xi^{\alpha}}
$$

The surface element at the reference surface is

$$
\mathrm{d} A=\sqrt{a} \mathrm{~d} \xi^{1} \mathrm{~d} \xi^{2} .
$$

The covariant components of the curvature tensor $\mathbf{b}=b_{\alpha \beta} \mathbf{a}^{\alpha} \otimes \mathbf{a}^{\beta}$,

$$
b_{\alpha \beta}=\mathbf{a}_{3} \cdot \mathbf{a}_{\alpha, \beta}=\mathbf{a}_{3} \cdot \mathbf{a}_{\beta, \alpha}=-\mathbf{a}_{\alpha} \cdot \mathbf{a}_{3, \beta}=-\mathbf{a}_{\beta} \cdot \mathbf{a}_{3, \alpha},
$$

offer an information about the change of normal vector $\mathbf{a}_{3}$. Their symmetry is a consequence of the interchangeability of second derivatives. The two rightmost expressions follow from the orthogonality condition $\mathbf{a}_{3} \cdot \mathbf{a}_{\alpha}=0$. It is a trivial consequence of (205) that

$$
\mathbf{a}_{3, \alpha}=-b_{\alpha \beta} \mathbf{a}^{\beta}=-b_{\alpha}^{\beta} \mathbf{a}_{\beta} .
$$

An analogous relation for the in-plane basis vectors,

$$
\mathbf{a}_{\beta, \alpha}=\Gamma_{\beta \alpha}^{\mu} \mathbf{a}_{\mu}+b_{\beta \alpha} \mathbf{a}_{3}, \quad \mathbf{a}^{\beta}{ }_{, \alpha}=-\Gamma_{\mu \alpha}^{\beta} \mathbf{a}^{\mu}+b_{\alpha}^{\beta} \mathbf{a}_{3},
$$

makes use of the surface Christoffel symbols,

$$
\Gamma_{\beta \alpha}^{\mu}=\mathbf{a}^{\mu} \cdot \mathbf{a}_{\beta, \alpha}=-\mathbf{a}_{\beta} \cdot \mathbf{a}^{\mu}{ }_{, \alpha}=\Gamma_{\alpha \beta}^{\mu} .
$$

The second equality is a consequence of $\mathbf{a}^{\mu} \cdot \mathbf{a}_{\beta}=\delta_{\beta}^{\mu}$. Equations (206) and (207) are known as the derivation rules of Weingarten and of Gauss, respectively. The covariant derivatives of any surface vector $\mathbf{y}=y^{\beta} \mathbf{a}_{\beta}=$ $y_{\beta} \mathbf{a}^{\beta}$ are

$$
\mathbf{a}^{\beta} \cdot \mathbf{y}_{, \alpha}=y_{, \alpha}^{\beta}+\Gamma_{\mu \alpha}^{\beta} y^{\mu}=\left.y^{\beta}\right|_{\alpha}, \quad \mathbf{a}_{\beta} \cdot \mathbf{y}_{, \alpha}=y_{\beta, \alpha}-\Gamma_{\beta \alpha}^{\mu} y_{\mu}=\left.y_{\beta}\right|_{\alpha} .
$$

In particular, the divergence of vector field $\mathbf{y}$ reads

$$
\operatorname{div} \mathbf{y}=\left.y^{\alpha}\right|_{\alpha}=y_{, \alpha}^{\alpha}+\Gamma_{\mu \alpha}^{\alpha} y^{\mu}=\frac{1}{\sqrt{a}}\left(\sqrt{a} y^{\alpha}\right)_{, \alpha}
$$

since $\Gamma_{\mu \alpha}^{\alpha}=\frac{1}{\sqrt{a}}(\sqrt{a})_{, \alpha}$ as can be confirmed by direct calculation. The surface divergence theorem will play a central role,

$$
\int_{\mathcal{A}} \frac{1}{\sqrt{a}}\left(\sqrt{a} y^{\alpha}\right)_{, \alpha} \mathrm{d} A=\int_{\partial \mathcal{A}} y^{\alpha} v_{\alpha} \mathrm{d} s
$$

converting the area integral into a path integral along the boundary $\partial \mathcal{A}$ of the surface $\mathcal{A}$ (or of a simply connected subset), where

$$
\mathrm{d} s=\sqrt{a_{\alpha \beta} \mathrm{d} \xi^{\alpha} \mathrm{d} \xi^{\beta}}
$$

is the line element on the curve $c\left(\xi^{1}, \xi^{2}\right)=0$, which represents $\partial \mathcal{A}$, and $\boldsymbol{v}=v_{\alpha} \mathbf{a}^{\alpha}$ is the corresponding outward unit normal vector.

For contravariant tensor components $y^{\mu \beta}=\mathbf{a}^{\mu} \cdot \mathbf{Y} \cdot \mathbf{a}^{\beta}$ the covariant derivatives are

$$
\mathbf{a}^{\mu} \cdot \mathbf{Y}_{, \alpha} \cdot \mathbf{a}^{\beta}=y_{, \alpha}^{\mu \beta}+\Gamma_{\nu \alpha}^{\beta} y^{\mu \nu}+\Gamma_{\nu \alpha}^{\mu} y^{\nu \beta}=\left.y^{\mu \beta}\right|_{\alpha} .
$$


The divergence of a tensor $\mathbf{Y}=y^{\mu \nu} \mathbf{a}_{\mu} \otimes \mathbf{a}_{v}$ is given by

$$
\operatorname{div} \mathbf{Y}=\left.y^{\mu \alpha}\right|_{\alpha} \mathbf{a}_{\mu}=\frac{1}{\sqrt{a}}\left(\sqrt{a} \mathbf{Y} \cdot \mathbf{a}^{\alpha}\right)_{, \alpha}
$$

with

$$
\left.y^{\mu \alpha}\right|_{\alpha}=y_{, \alpha}^{\mu \alpha}+\Gamma_{\nu \alpha}^{\alpha} y^{\mu \nu}+\Gamma_{\beta \alpha}^{\mu} y^{\beta \alpha}=\frac{1}{\sqrt{a}}\left(\sqrt{a} y^{\mu \alpha}\right)_{, \alpha}+\Gamma_{\beta \alpha}^{\mu} y^{\beta \alpha},
$$

and the tensor divergence theorem reads

$$
\int_{\mathcal{A}} \frac{1}{\sqrt{a}}\left(\sqrt{a} \mathbf{Y} \cdot \mathbf{a}^{\alpha}\right)_{, \alpha} \mathrm{d} A=\int_{\partial \mathcal{A}} \mathbf{Y} \cdot \boldsymbol{v} \mathrm{d} s .
$$

It can be verified by direct calculation that

$$
\left.a^{\alpha \beta}\right|_{\mu}=0,\left.\quad a_{\alpha \beta}\right|_{\mu}=0,\left.\quad \delta_{\beta}^{\alpha}\right|_{\mu}=0
$$

(Ricci lemma), and similarly

$$
\left.\epsilon^{\alpha \beta}\right|_{\mu}=0,\left.\quad \epsilon_{\alpha \beta}\right|_{\mu}=0
$$

\section{References}

1. Basar, Y., Krätzig, W.B.: Mechanik der Flächentragwerke. Springer, Wiesbaden (1985)

2. Basar, Y., Krätzig, W.B.: A consistent shell theory for finite deformations. Acta Mech. 76, $73-87$ (1989)

3. Chapelle, D., Bathe, K.J.: The Finite Element Analysis of Shells: Fundamentals. Computational Fluid and Solid Mechanics, 2nd edn. Springer, Berlin (2011)

4. Chróścielewski, J., Makowski, J., Stumpf, H.: Genuinely resultant shell finite elements accounting for geometric and material non-linearity. Int. J. Numer. Methods Eng. 35, 63-94 (1992)

5. Chróścielewski, J., Witkowski, W.: Four-node semi-EAS element in six-field nonlinear theory of shells. Int. J. Numer. Methods Eng. 68, 1137-1179 (2006)

6. Ciarlet, P.C.: Mathematical Elasticity. Vol. 3: Theory of Shells. Studies in Mathematics and its Applications. Elsevier, Amsterdam (2000)

7. Ciarlet, P.G.: Mathematical Elasticity. Vol. 2: Theory of Plates. Studies in Mathematics and its Applications. Elsevier, Amsterdam (1997)

8. Ciarlet, P.G.: An Introduction to Differential Geometry with Applications to Elasticity. Springer, Dordrecht (2005)

9. Eliseev, V.V., Vetyukov, Y.M.: Finite deformation of thin shells in the context of analytical mechanics of material surfaces. Acta Mech. 209, 43-57 (2010)

10. Fox, D.D., Simo, J.C.: A drill rotation formulation for geometrically exact shells. Comput. Methods Appl. Mech. Eng. 98, 329-343 (1992)

11. Frostig, Y., Kardomateas, G.A., Rodcheuy, N.: Non-linear response of curved sandwich panels-extended high-order approach. Int. J. Non-Linear Mech. 81, 177-196 (2016)

12. Green, A.E., Zerna, W.: Theoretical Elasticity, 1st edn. University Press, Oxford (1954)

13. Gruttmann, F., Stein, E., Wriggers, P.: Theory and numerics of thin shells with finite rotations. Ingenieur Arch. 59, 54-67 (1989)

14. Haupt, P.: Continuum Mechanics and Theory of Materials, 2nd edn. Springer, Berlin (2002)

15. Ibrahimbegović, A.: Stress resultant geometrically nonlinear shell theory with drilling rotations-Part I. A consistent formulation. Comput. Methods Appl. Mech. Eng. 118, 265-284 (1994)

16. Ibrahimbegović, A.: Stress resultant geometrically nonlinear shell theory with drilling rotations-Part II. Computational aspects. Comput. Methods Appl. Mech. Eng. 118, 285-308 (1994)

17. Kienzler, R.: On consistent plate theories. Arch. Appl. Mech. 72, 229-247 (2002)

18. Kienzler, R., Schneider, P.: Second-order linear plate theories: partial differential equations, stress resultants and displacements. Int. J. Solids Struct. 115, 14-26 (2017)

19. Libai, A., Simmonds, J.G.: Nonlinear elastic shell theory. In: Hutchinson, J.W., Wu, T.Y. (eds.) Advances in Applied Mechanics, vol. 23, pp. 272-371. Academic Press, New York (1983)

20. Mindlin, R.: Influence of rotary inertia and shear on flexural motion of isotropic elastic plates. ASME J. Appl. Mech. 18, 31-38 (1951)

21. Naghdi, P.M.: The theory of shells and plates. In: Flügge, S., Truesdell, C. (eds.) Handbuch der Physik VIa/2, pp. 425-640. Springer, Berlin (1972)

22. Neff, P., Hong, K.I., Jeong, J.: The Reissner-Mindlin plate is the $\Gamma$-limit of Cosserat elasticity. Math. Models Methods Appl. Sci. 20, 1553-1590 (2010)

23. Reddy, J.N.: A general non-linear third-order theory of plates with moderate thickness. Int. J. Non-Linear Mech. 25, 677-686 (1990) 
24. Reissner, E.: The effect of transverse shear deformation on the bending of elastic plates. ASME J. Appl. Mech. 12, A69-A77 (1945)

25. Reissner, E.: On consistent first approximations in the general linear theory of thin elastic shells. Ingenieur Arch. 40, 402-419 (1971)

26. Ricci-Curbastro, G., Levi-Civita, T.: Méthodes de calcul différentiel absolu et leurs applications. Math. Ann. 54, 125-201 (1901)

27. Rössle, A., Bischoff, M., Wendland, W., Ramm, E.: On the mathematical foundation of the 1-1-2 plate model. Int. J. Solids Struct. 36, 2143-2168 (1998)

28. Schneider, P., Kienzler, R.: Comparison of various linear plate theories in the light of a consistent second-order approximation. Math. Mech. Solids 20, 871-882 (2015)

29. Schnell, W., Eschenauer, H.: Elastizitätstheorie II: Schalen. Bibliographisches Institut, Mannheim (1984)

30. Simmonds, J.G.: A Brief on Tensor Analysis. Undergraduate Texts in Mathematics, 2nd edn. Springer, New York (1994)

31. Simo, J.C., Fox, D.D.: On a stress resultant geometrically exact shell model. Part I: Formulation and optimal parametrization. Comput. Methods Appl. Mech. Eng. 72, 276-304 (1989)

32. Simo, J.C., Fox, D.D., Rifai, M.S.: On a stress resultant geometrically exact shell model. Part III: Computational aspects of the nonlinear theory. Comput. Methods Appl. Mech. Eng. 79, 21-70 (1990)

33. Simo, J.C., Rifai, M.S., Fox, D.: On a stress resultant geometrically exact shell model. Part IV: Variable thickness shells with through-the-thickness stretching. Comput. Methods Appl. Mech. Eng. 81, 91-126 (1990)

34. Steigmann, D.J.: Two-dimensional models for the combined bending and stretching of plates and shells based on threedimensional linear elasticity. Int. J. Eng. Sci. 46, 654-676 (2008)

35. Vekua, I.N.: Shell Theory: General Methods of Construction (translated from the Russian by T. Gabeskiria). Vol. 25 of Monographs, Advanced Texts, and Surveys in Pure and Applied Mathematics. Pitman Advanced Publishing Program, Boston (1985)

36. Vetyukov, Y., Kuzin, A., Krommer, M.: Asymptotic splitting in the three-dimensional problem of elasticity for nonhomogeneous piezoelectric plates. Int. J. Solids Struct. 48, 12-23 (2011)

37. Wagner, W., Gruttmann, F.: A robust non-linear mixed hybrid quadrilateral shell element. Int. J. Numer. Methods Eng. 64, 635-666 (2005)

38. Wempner, G., Talaslidis, D.: Mechanics of Solids and Shells. CRC Press, Boca Raton (2003)

39. Wiśniewski, K.: A shell theory with independent rotations for relaxed Biot stress and right stretch strain. Comput. Mech. 21, 101-122 (1998)

40. Wiśniewski, K.: Finite Rotation Shells: Basic Equations and Finite Elements for Reissner Kinematics. Lecture Notes on Numerical Methods in Engineering and Sciences. Springer, Berlin (2010)

41. Wiśniewski, K., Turska, E.: Four-node mixed Hu-Washizu shell element with drilling rotation. Int. J. Numer. Methods Eng. 90, 506-536 (2012)

Publisher's Note Springer Nature remains neutral with regard to jurisdictional claims in published maps and institutional affiliations. 